Review Article

\title{
Structure-Function Relationships behind the Phenomenon of Cognitive Resilience in Neurology: Insights for Neuroscience and Medicine
}

\author{
David Rudrauf \\ Laboratory of Functional Imaging (LIF), INSERM U678S, 91 Boulevard de l'Hôpital, 75013 Paris, France \\ Correspondence should be addressed to David Rudrauf; david.rudrauf@gmail.com
}

Received 2 December 2013; Revised 7 April 2014; Accepted 8 April 2014; Published 4 August 2014

Academic Editor: Jan Gläscher

Copyright (c) 2014 David Rudrauf. This is an open access article distributed under the Creative Commons Attribution License, which permits unrestricted use, distribution, and reproduction in any medium, provided the original work is properly cited.

\begin{abstract}
The phenomenon of cognitive resilience, that is, the dynamical preservation of normal functions despite neurological disorders, demonstrates that cognition can be highly robust to devastating brain injury. Here, cognitive resilience is considered across a range of neurological conditions. Simple computational models of structure-function relationships are used to discuss hypotheses about the neural mechanisms of resilience. Resilience expresses functional redundancies in brain networks and suggests a process of dynamic rerouting of brain signals. This process is underlined by a global renormalization of effective connectivity, capable of restoring information transfer between spared brain structures via alternate pathways. Local mechanisms of synaptic plasticity mediate the renormalization at the lowest level of implementation, but it is also driven by top-down cognition, with a key role of self-awareness in fostering resilience. The presence of abstraction layers in brain computation and networking is hypothesized to account for the renormalization process. Future research directions and challenges are discussed regarding the understanding and control of resilience based on multimodal neuroimaging and computational neuroscience. The study of resilience will illuminate ways by which the brain can overcome adversity and help inform prevention and treatment strategies. It is relevant to combating the negative neuropsychological impact of aging and fostering cognitive enhancement.
\end{abstract}

\section{Introduction}

In neurology, one is often faced with a relative disconnect between the clinical presentation and the underlying neuropathology or amount of brain damage [1-10]. One observes cognitive functions that appear to be relatively preserved in spite of damage to brain systems that one would expect to be normally implicated in these functions. Patients with similar brain damage or neurological disorder often show quite different neuropsychological profiles, with different evolutions, and cliniconeuropathological relationships are characterized by a strong between-subject variability $[2,9]$.

Part of this between-subject variability is underlined by static intrinsic differences in structure-function relationships in different subjects, for example, the lateralization of language, which makes certain patients less susceptible than others to certain impairments for similar brain damage, for example, damage to the left hemisphere, in which many critical functions for language are most often implemented in humans. Part of the variability is related to processes of recovery that are more or less efficient across individuals following similar initial impairments due to similar brain damage. But more generally, as reviewed below, quite often patients with severe, extensive, irreversible bilateral brain damage show preserved functions or eventually recover in full or in part over time in a dynamic manner from brain damage causing initial cognitive impairments, sometimes very rapidly, often after a long and painful struggle. Thus, in cases in which brain structures cannot be rebuilt through structural plasticity, there are within-subject changes in structure-function relationships manifested in the recovery of cognition.

In other words, there is a phenomenon of "cognitive resilience" to brain damage. Resilience, generally speaking, is the ability of a system to resist dynamically a perturbation or adverse condition that challenges the integrity of its 
normal operation and to preserve function as a result in reference to some initial design or normative functional standards. The concept of resiliency is relevant to and employed in many scientific domains, including material science, biology, ecology, economy, computer and network science, psychiatry, and neurology. In computer networking, which is relevant here: "resilience is the ability of the network to provide and maintain an acceptable level of service in the face of various faults and challenges to normal operation" (see ResiliNets initiative: https://wiki.ittc.ku.edu/resilinets). It features disruption tolerance; that is, "the ability of a system to tolerate disruptions in connectivity among its components", and fault tolerance against failure, that is, "the occurrence of an event in which an entity does not meet its in-service functional and performance requirements or expectations" (see https://wiki.ittc.ku.edu/resilinets_wiki). According to this model (see ResiliNets Architecture), which has broad applications, resilient systems will integrate expectations about the inevitability of fault, understand (or possess a model of) normal operations, expect adverse events and respond to adverse events and conditions. Resilience can build upon a strategy involving detection, defense, remediation and recovery mechanisms, based on a control loop.

In the context of the present paper, I am discussing the resilience of cognition despite brain damage that results in extensive and irreversible destruction and disconnection of the structural backbone of the brain. In neurological resilience, the overall architecture of information processing and control that underlies normal cognition appears to resist the structural perturbations that challenge its integrity. There is an apparent decoupling between structure and function, in relation to normative anatomical expectations or previous structure-function relationships, which allows the brain to recover function in a manner that is compatible with normal functional or cognitive standards.

The phenomenon of cognitive resiliency is pervasive and observed across a large spectrum of neurological disorders. It is present in cases of focal and stable brain damage-even in dramatic cases of very extensive lesions (e.g., [2, 3]). Cognitive resilience is also found in cases of diffuse brain damage, both with stable lesions, for example, following traumatic brain injury [4], as well as progressive lesions, for example, neurodegenerative processes such as Alzheimer's disease [11]. Likewise it is found in dramatic developmental cases [10]. It is difficult to quantify, and the interindividual variability it introduces in neurological populations complicates our ability to understand and model neurological conditions with specificity (and thus approach treatments). It challenges the central aim of maximizing sensitivity and specificity in diagnostics, prognostics, treatment planning, treatment outcome monitoring, and more generally neurological research.

Theoretical rationale and empirical evidence $[2,3,8$, 12-15] suggest that cognitive resilience may, in part, take advantage of the existence of processing resources that are distributed across large portions of the brain and which are highly redundant. It builds upon the possibility of exploiting alternative pathways, which can be direct or indirect, for restoring information transfer and maintain cognitive functions (e.g., [13]). The notion of resilience is related to that of "cognitive reserve" [8], an innate and acquired "reserve" of compensatory mechanisms, which can support resilience as a phenomenon and which notably appears to be correlated with premorbid, higher-order cognitive skills.

Resilience notably concerns "higher-order cognition," which entails an ensemble of psychological mechanisms and phenomena including executive functions, attention, general intelligence, and self-awareness. These form, together, a coherent functional whole that normally constitutes the core of a wakeful and willful mind in the process of conscious perception, imagination, decision-making, and action planning $[12,16,17]$. "Higher-order cognition" itself appears to be generally more resilient to damage than more specialized, modal forms of cognition, such as basic vision and audition $[1,12,18,19]$.

In this paper, I argue that beyond reflecting sheer redundancies in the brain, the phenomenon of resilience manifests degrees of freedom in anatomofunctional relationships, which in turn challenge to some extent the standard anatomofunctional framework as it traditionally supposes a strong and direct relationship between structure and function. It therefore represents a methodological challenge for functional neuroanatomy and research on biomarkers from multimodal neuroimaging.

I argue that resilience manifests mechanisms of dynamic rerouting of information streams in the brain that are necessary to maintain functional integrity in face of network structural failure. I propose a framework for understanding, modeling, and quantifying resilience in this perspective. I also argue that resilience can provide a paradigm for approaching fundamental questions about mind-brain relationships. It suggests provocative hypotheses about the mechanisms and principles underlying brain computation, including the possibility that some integrative aspects of cognition relate to the brain according to principles that are analogous to those underlying virtualization in computer and network science. It thus reopens the question of the neural code.

In the following, I first consider the phenomenon of cognitive resilience in the context of various neurological disorders, from stable focal brain damage-with an example of preserved higher-order cognition following extensive bilateral damage - to stable diffuse brain damage, using the example of disorders of consciousness, and progressive diffuse brain damage, exemplified by Alzheimer's disease. I briefly consider resilience to developmental aberrations such as hydrocephaly. I then discuss hypotheses regarding the relationships between resilience, distributed processing, and anatomofunctional dissociations. I also discuss the corollary hypothesis that the mind might relate to the brain through a pile of layers of abstraction and a process of virtualization (in the sense that these phrases have in computer and network science). I consider possible research directions and highlights challenges for understanding and controlling resilience based on multimodal neuroimaging and computational neuroscience. I conclude that resilience offers new venues for clinical and basic research, in particular with regard to preventive and clinical medicine, but also for the mitigation of aging and cognitive enhancement. 


\section{The Phenomenon of Cognitive Resilience to Neurological Disorders}

2.1. Resilience to Developmental Aberrations: Setting the Stage. One of the most striking illustrations of the resilience of normal cognition to the presence of severely abnormal brain structures can be found in the literature on abnormal development (see [10]), which I will only briefly mention here. This literature challenges how one thinks about anatomical-functional relationships and illustrates how extreme resilience can be in certain rare developmental cases.

While genetic and environmental factors can result in extremely aberrant brain development (with dramatic morphological and structural alterations), in many cases, there is often a relative preservation of core mental abilities [10]. In a rather extreme example, a 44-year-old civil servant and father of two with relatively low intelligence, but normal social functioning, was found to have preserved mental abilities despite significant ventricular enlargement and a very thin cortical mantle due to a history of hydrocephaly [20] (Figure 1(a)). While the processes underlying neuroanatomical reorganization and recovery after brain damage remain unclear, developmental cases suggest that extremely different brains across individuals can support normal cognition. Furthermore, it is textbook knowledge and common clinical observation that age plays a critical role in resilience, since early in development when the potential for brain plasticity is highest resilience can be greater.

2.2. Resilience to Stable Focal Brain Damage. Stable, focal brain damage, which is damage encompassed by a continuous and unique lesion which can nevertheless be extensive and bilateral, can be caused by a variety of etiologies and mechanisms, ranging from ischemic or hemorrhagic strokes to surgery of tumors or epileptic foci and certain types of encephalitis, such as herpes simplex virus encephalitis. These sources of brain damage constitute the most common basis for the human lesion method ([21]; see [22, 23]).

It is well established that many higher cognitive functions do not appear to be completely and systematically disrupted by focal brain damage to the cerebral cortex $[1,2,15,19,24]$. In contrast, such damage can cause systematic and durable impairments in more specialized aspects of cognition, in particular in sensorimotor processing, for example, vision and audition. Impairments can also affect speech and language, for example, aphasia, or memory-laden functions, for example, the case of anterograde amnesia, which is quite a systematic syndrome following bilateral damage to the medial temporal lobe. These disorders are also susceptible to relatively systematic and sometimes permanent impairments. Working memory, decision-making, attention, and other executive functions, for example, the frontal syndromes of perseveration, may also be impaired and may engender a loss of autonomy to various degrees, more or less systematically and persistently, in particular when damage is bilateral [19, 25]. Such impairments are thought to be caused either by the disruption of modular processing in functionally specialized, cortical, and subcortical regions, or by the dissection of fiber tracts, causing disconnection syndromes (see $[23,26])$. But overall: "one sided removal of, or injury to, any area of the cerebral cortex does not abolish conscious thinking. It may change the content of awareness, interfere with voluntary acts, render less effective planned action, and deprive the patient of word symbols-but he still thinks and weeps, perhaps, at his own pitiful incapacity" [18]. Moreover, except in specific cases, higher-order cognition in its core remains generally quite robust even to extensive and bilateral focal brain damage, or recovers sometimes quite fast after the acute phase following damage onset. A striking illustration of resilience of higher-order cognition to extensive bilateral brain damage can be found in neurological cases that challenge contemporary knowledge and hypotheses about the neural substrate of self-awareness (SA).

Over the past decade, an ever-growing body of functional neuroimaging research has explored the neural basis of SA. For instance, largely based on such findings, it has recently been proposed that the anterior insula (AI) represents one of the most critical and essential substrates underlying SA in humans [27-30]. In addition, the medial prefrontal cortex (mPFC) has been proposed to be essential for self-related processing [31-34]. These hypotheses predict that damage to these regions should disrupt, or perhaps even abolish essential aspects of SA. However, an emerging body of work in a rare patient with extensive bilateral damage encompassing both the AI and $\mathrm{mPFC}$ directly contradicts this prediction $[3,13,35]$.

The patient, Roger, is a male in his late 50s with a college education whose brain was severely damaged in 1980 as a result of herpes simplex encephalitis (Figure 1(b)). His brain damage is bilateral, more extensive on the right, and encompasses the insular cortex, the anterior cingulate cortex (ACC), and the mPFC. Moreover the damage extends to the basal forebrain and the entire medial temporal lobe, including the amygdala and hippocampus bilaterally. In addition, the white matter is extensively damaged, in particular on the right, extending from the frontal and temporal poles to the anterior inferior parietal lobule. The damage spares the brainstem, cerebellum, thalamus, hypothalamus, basal ganglia, and most posterior cortical structures in the occipital and parietal lobes.

Studies from our group have demonstrated that Roger has largely intact cognitive abilities with normal intelligence, language, and even executive functioning [35]. Roger exhibits an overall normal neuropsychological profile except for a profound anterograde amnesia and a temporally graded retrograde amnesia that is most apparent during the 10 years preceding the onset of his brain damage.

Moreover, Roger has preserved interoceptive awareness of cardiac sensations [13]. We used a double-blind, randomized, pharmacological protocol involving the infusion of Isoproterenol-a Beta-adrenergic agonist, which provokes well-titrated, transient increases in heart rate and heart contraction. The results supported the hypothesis that interoceptive awareness is an integrative and synthetic phenomenon that relies on multiple pathways, including somatosensory pathways. The latter are traditionally conceived as transducing and carrying exteroceptive signals. The study suggested that these pathways constitute a sufficient channel to carry 


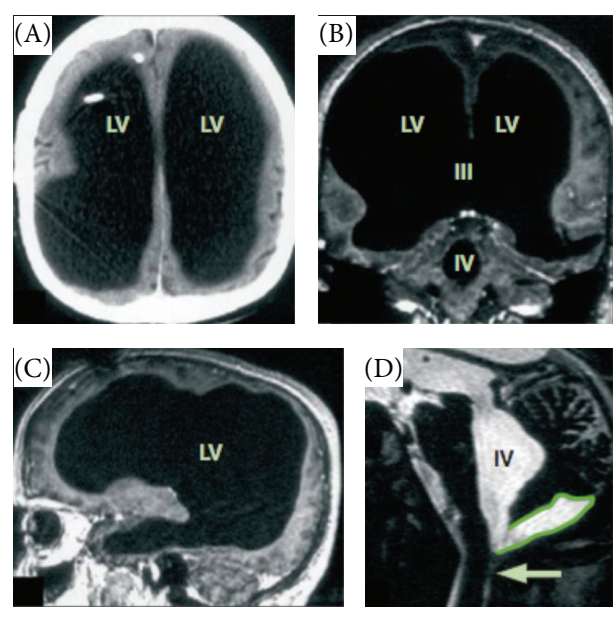

(a)
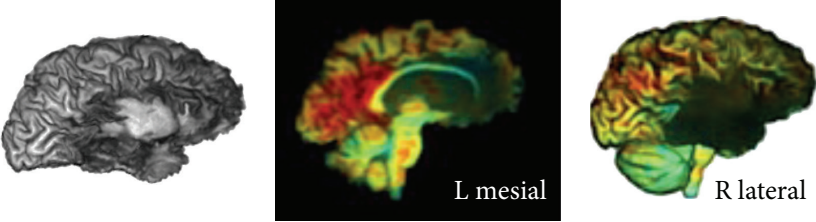

(b)

FIGURE 1: Examples of resilience to brain damage. (a) A 44-year-old civil servant and father of two with relatively low intelligence, but normal social functioning, was found to have preserved mental abilities despite significant ventricular enlargement and a very thin cortical mantle due to a history of hydrocephaly [20]. (b) Roger: patient with bilateral destruction of the limbic system following herpes simplex virus encephalitis. Contrary to all expectations, the patient presents largely preserved cognition and self-awareness (adapted from [3]).

peripheral visceral information to the brain for an integration that cannot be performed at the level of the insula in this patient.

We also demonstrated that Roger's SA was largely preserved, at all levels of its functional hierarchy, from core, to extended and reflective SA (see $[3,12]$ ), using a battery of tests targeting SA at multiple levels (mirror test, self-recognition from pictures, self-agency tasks, personality ratings, and selfawareness questionnaire). Furthermore, functional connectivity analyses from resting state fMRI collected in Roger suggested that the dynamics of interaction among preserved sectors of his brain, including preserved medial parietal regions of the so-called default mode network (see [36]), could contribute to support Roger's preserved functions with regard to SA (Figure 1(b)).

In conclusion, Roger is a conscious, self-aware, and sentient human being despite the destruction of nearly onethird of his brain, including cortical centers presumed to play a critical role in SA according to some prominent hypotheses in the literature. His general appreciation of his memory deficit indicates that he has some awareness of his cognitive limitations. However due to Roger's pervasive amnesia, his autobiographical SA, especially with regard to his autobiographical self [19], that is, his own representations and narratives of himself built upon autobiographical memory, appears to be largely limited to the repositories of stored episodic and semantic knowledge consolidated during his childhood and young adult life.

This is not a completely isolated case. Other herpes simplex encephalitis patients, with brain damage almost identical to Roger's, and even more extensive, have been described [37] but had never been presented as showing any evidence for defective SA ([19], p. 117-121). A retrospective report following up on Philippi et al. [3], with a focus on emotion and sentience, found indications of similarly preserved functions in such herpes simplex encephalitis patients [38]. In such patients, emotions, from basic to social emotions, from experience to recognition, also appear more preserved than what could be expected from their damage to regions of the brain supposed to be critical for emotions, including the amygdala, anterior cingulate cortex (ACC), and insular cortex [38-40].

SA also appears to remain largely preserved in neuropsychological cases of patients with profound retrograde and anterograde amnesia [19], including patients with extreme amnesia such as Clive Wearing [41]. Interestingly, the patient demonstrated intact Core Consciousness ([19]; see also [12]), that is, the core, preattentive private experience and feeling of being a willful agent owner of his own thoughts and at least minimally aware of himself as such. The patient also had remaining controlled, self-reflective abilities pertaining to SA. They were expressed in distressful statements like: "I'm alive for the very first time." He could remember much of his previous self and had in recent years shown an ability to integrate an awareness of his memory deficit.

\subsection{Resilience to Stable Diffuse Brain Damage: The Case} of Disorders of Consciousness. Recent research in patients with disorders of consciousness (DOC), such as persistent vegetative states (PVS) or minimally conscious state (MCS) patients, can further provide some surprising support for the notion that many higher-order cognitive functions are highly resilient to neuropathological insults. This is expressed clinically through a within-subject process of recovery of function, often accompanied with daily fluctuations in mental status and marked by an important between-subject variability in capacity for resilience $[4,5,42,43]$.

Contrary to coma, which generally arises from damage to the upper brainstem's Ascending Reticular Activating System (ARAS), in PVS and MCS there is sufficient diencephalic 
and upper brainstem preservation of function for supporting basic sleep-wake cycles, primitive orienting responses, and basic brainstem and spinal reflexes. In PVS, cognitive functions, sensory modalities, speech, and movement are dramatically interrupted, and the patients are entirely noncommunicative. In MCS, there can be rare and intermittent signs of following simple commands, occasional yes/no responses and verbalizations, and congruent basic affective responses. From a pathological standpoint, PVS generally results from very diffuse necrosis of the cerebral cortex and/or diffuse shear injury of axons in the thalamocortical system, and/or damage to the thalamic intralaminar nuclei. PVS can occur following traumatic brain injury (TBI), metabolic failure (e.g., anoxia), and poisoning, as well as massive or diffuse strokes. MCS is caused by a similar etiology and pathology, with damage however often less diffuse topographically. Overall, the metabolic rate of glucose in PVS and MCS ranges between $40 \%$ and $50 \%$ of a normal wakeful brain, and it is comparable with that of coma and general anesthesia (see [44]).

Apart from cases of focal brain damage that affect subcortical regions of the thalamus, basal ganglia, or brainstem, many PVS cases suggest that consciousness may only disappear whenever extensive, diffuse, bilateral damage disrupts much of the thalamocortical system and largescale functional interactions between brain regions $[45,46]$. Nevertheless, even in such circumstances, evidence suggests that residual consciousness can be present in some PVS patients $[4-6,44]$.

In a series of studies $[5,6]$, several PVS patients were able to perform mental imagery of playing tennis or walking through different rooms, following verbal cues. Playing tennis is reliably associated with activation of the supplementary motor area (SMA) and navigating rooms is reliably associated with activation of the parahippocampal gyrus, posterior parietal lobe, and premotor cortex. While instructed to perform the task, the patient activated the exact same regions as predicted based on data collected in normal participants, in accordance with the instructions. Furthermore, in a follow-up experiment, one of the patients was asked a few biographical questions [6]. The patient was instructed to perform one or the other type of mental imagery depending on whether the answer to the questions was "yes" or "no." As measured by the activation of the target regions, the "answers" were $100 \%$ correct. Further neuroanatomical investigations will be essential to establish whether the integrity of certain regions of the thalamocortical system (e.g., regions of the default mode network (DMN) that are discussed below), is more critical than that of other regions for PVS cases to demonstrate such preserved covert consciousness.

More generally, resilience is also manifested in the impressive capacity of many patients to recover from coma, PVS, and MCS [47], and this may depend on the details of the anatomy of the damage. In Giacino and Kalmar's study [48], up to seventy-three percent of PVS patients showing early signs of intact visual pursuit were able to recover other clear-cut signs of consciousness by 12 months. Fifty percent of MCS patients and three percent of PVS patients had "no disability" to "moderate disability" at 1-year post injury.
2.4. Resilience to Progressive Diffuse Brain Damage: The Case of Alzheimer's Disease. In addition to the aforementioned neurological cases of DOC, neurodegenerative diseases, such as Alzheimer's disease (AD) and frontotemporal dementia (FTD), represent further examples of the clinical manifestation of within-subject cognitive resilience, with pronounced fluctuations in mental status and a strong between-subject variability, following this time a progressively aggravating, irreversible distributed neuropathology affecting both structure and function [8].

Understanding the specific mechanisms associated with resilience to $\mathrm{AD}$ is an important clinical, financial, and societal challenge for public health, because $\mathrm{AD}$ is turning into an epidemic among the aging population. This issue strongly affects AD care-burden management, prognosis, the development of strategies of rehabilitation, treatment planning and management, and more generally behavioral neurology. There is currently no known treatment for AD. According to the 2010 World Alzheimer report, about 35.6 million people in the world struggle with dementia, representing an annual cost of more than US $\$ 600$ billion. The prospect is to see the incidence of the disease double over the next 20 years.

AD is a "dual clinicopathological entity" (see [9]) that is characterized by a neurodegenerative process [49]. It presents a typical pattern of progression [50], including the following.

(1) A clinical phenotype, which entails progressive dementia following a prodromal phase, often developing over decades, with quite variable rates of decline across individuals. Typically, symptomatic phase starts with episodic memory impairments and is followed by anomia, disorders of controlled and executive process, appearance of prevalent spatial cognition impairments, and emotional disorders (e.g., apathy, panic attacks), personality changes, and impaired judgment and consciousness, progressing into a complete breakdown of the mind. Anosognosia, a reduced self-awareness regarding one's own deficits [51], is also a prevalent symptom in $\mathrm{AD}$ [52] that can lead to unsafe behaviors [53], neuropsychiatric comorbidities, including apathy and panic attacks [54, 55]. Moreover, it represents a strong burden for caregivers and has deleterious effects on quality of the patient's life [56].

(2) A progressive pattern of neuropathological alterations [57], which seemingly propagates along neural pathways $[58,59]$. Senile plaques (often presymptomatic and weakly correlated with clinical expression) are associated with extracellular $\beta$-amyloid $(\mathrm{A} \beta)$ aggregates. Neurofibrillary tangles (more strongly correlated with clinical expression) are associated with intracellular tau-amyloid fibril aggregates. A progressive pattern of diffuse atrophy [57] and abnormal resting metabolism [60] develops consecutively with collateral oxidative and inflammatory stress, synaptic loss and neuronal loss, especially in association cortices. Damage starts with mesial-temporal lobe structures (medial temporal atrophy is one of 
the biomarkers most associated with cognitive decline [57]) and progresses with temporal, parietal, and frontal atrophy, while also affecting many subcortical structures $[61,62]$.

Several variants of the disease exist, and its etiology can be idiopathic or genetic, in particular in rare cases of early-onset manifestations of the disease. Multiple demographic and functional factors contribute either to mitigate or aggravate the risk of disease. (http://www.alz.co.uk/research/worldreport).

There is a growing consensus [63-68], on both clinical and neuropathological grounds, that $\mathrm{AD}$ is a disconnection syndrome [26], with clinical expression in impaired crossmodal integration. A progressive breakdown of communication between brain systems at the large scale is observed and is reflected in both structural and functional connectivity (see Definitions). It affects both interhemispheric and intrahemispheric interactions, notably in frontoparietal, parietotemporal, and temporofrontal networks that are all affected by $\mathrm{AD}$ pathology. Fiber tracts such as the cingulum, the superior longitudinal fasciculus, uncinate fasciculus, corpus callosum, and most association tracts [69] are affected. Beyond the sheer loss of neurons, the global organization and hierarchy of normal brain networks break down, and random patterns of connectivity replace the so-called "small-world" organization of normal brain networks, in which specialized subnetworks with dense intrinsic connections are well connected together at the large scale but at low wiring cost. The central "hubs" of the networks appear to undergo reorganization, that is, the regions characterized by the highest degree or bandwidth of connectivity with the rest of the network, from both a structural and functional standpoint. These hubs typically include midline structures such as the precuneus and medial prefrontal cortex. Global and local processing efficiency cannot be maintained in the context of shrinking processing resources and routes to connect them. As a result integrative and specialized functional processes cannot carry on the task of supporting normal cognition, and the mind as an integrated functional whole collapses.

Within this overall pathological process, the neuropsychological dependence of manifestations of cognitive impairments on the amount of brain alterations appears to be marked by a substantial interindividual variability [8, 49]. Full pathologic criteria for $\mathrm{AD}$ have been observed post mortem in $25 \%$ to $67 \%$ of the brains of elderly individuals with no indication of cognitive impairment prior to death [9, 70-72]. Certain AD patients show increased functional connectivity within frontal sectors as well as between frontal, thalamic, and striatal regions, which in turn suggests the presence of compensatory mechanisms aimed at coping with large-scale disconnection [67]. Generally speaking, in AD, variability across individuals in cerebral metabolic patterns is at least partly related to strategies of compensations aiming at optimizing the efficacy of key processes in a context of progressive decimation of resources [66].

Intriguingly, resilience in $\mathrm{AD}$ appears to be partly mediated by variables such as general intelligence, degree of literacy, educational attainment, occupational complexity, interpersonal skills, integration in social networks, personality variables, and leisure activity. These are all aspects of what has been referred to as "cognitive reserve" (CR) (see [8]). In other words, higher-order cognition and behaviors interact with the capacity for resilience. Given a comparable amount of brain damage, individuals who score higher in such variables tend to present with better cognitive performance and social adjustment, and also tend to be more easily managed by healthcare professionals, than individuals with lower CR. Furthermore, individuals with lower CR demonstrated a relative risk of developing dementia that was between 2.2 and 2.85 higher than individuals with higher CR [73]. In a meta-analysis, higher CR was associated with a lowered risk for incident dementia with summary odds ratio of 0.54 , and increased complex mental activity in late life was associated with lower dementia rates independent of other predictors [74].

Likewise, individuals with $\mathrm{AD}$ pathology and higher $\mathrm{CR}$ tend to present a point of inflexion in the progression of the disease, which marks the transition from a prodromal phase to dementia, which occurs at a more advanced stage of brain pathology [75] than individuals with low CR. Biomarkers of $\mathrm{AD}$ pathology, such as the uptake of ${ }^{11 \mathrm{C}} \mathrm{PiB}$ in positron emission tomography (PET) interacts with the number of years of education in predicting cognitive performance on scales such as the Clinical Dementia Rating sum of boxes, Mini-Mental State Examination, and Short Blessed Test [76]. Evidence also suggests that the relation between senile plaques and level of cognitive function differs by years of formal education [77]. Moreover, it has been shown that there is a relationship between higher CR and lower incidence or severity of anosognosia in dementia [78]. In other words, individuals with higher CR tend to be more resilient.

\section{Hypotheses about the Mechanisms of Cognitive Resilience}

As reviewed above, a wealth of clinical facts demonstrates that brains can preserve core aspects of the functional architecture of information processing that sustains higherorder cognition in spite of substantial structural damage causing irreversible losses of neuronal ensembles and systems of wires at multiple scales. Here I discuss a set of coherent hypotheses, often reasoning based on postulates, in order to explore possible explanations. Alternative explanations not envisioned in this paper probably exist. I am only proposing one possible conceptual approach, which I believe offers at this point the best overall explanatory power. As it will be apparent, some of those ideas are mainstream and others are more idiosyncratic and speculative, but I believe warrant attention. I frame the rationale and discussion by adopting a perspective on neural computation aimed at neural engineering.

3.1. Limits of Structural Plasticity for Recovery of Function. The brain is constituted by populations of neurons and glial cells distributed over many different anatomical locations. They are locally connected through intrinsic networks and at 
the large-scale through the white matter via neuronal bundles of fibers containing axons. At all scales, synapses mediate the functional interactions between connected neurons via fast electrochemical signaling. The integrity of structural connections, for example, long-range fiber bundles, is critical for many aspects of normal cognition. Our group has shown for instance that sectors of the white matter which contain large association fiber bundles connecting fronto-parietal systems play a causal role in general intelligence [79].

However, with resilience, functions are preserved or recover in spite of structural damage to gray matter regions and/or fiber tracts normally implicated in these functions. This process implies some form of anatomofunctional "plasticity."

Structural plasticity, for example, axonal regeneration, and synaptic and dendritic sprouting, can contribute to such plasticity [80]. However, lesions observed in the neurological cases considered here are too extended and severe for being compensated by a direct, large-scale physical rewiring of the entire system based on structural plasticity that would rebuild the original anatomical structures.

Thus structural plasticity alone cannot account for the anatomofunctional adaptations that appear necessary to support and account for the phenomenon of resilience.

Central tendencies in the relationships between structure and function, for example, anatomical location and taskrelated responses, which are observed in normal brains may appear integral to how the brain implements cognition. But these central tendencies are to some degree contingent, and their regularity often masks a strong interindividual variability in actual structure-function relationships. Such regularities should not be confused with an actual, strong, and rigid dependency between specific structures and specific functions [2]. The brain may have many alternative solutions to cognition built-in, which are revealed and manifested via neurological cases of resilience.

If large-scale structural plasticity alone cannot account for cognitive resilience, then anatomofunctional relationships, or more generally structure-function relationships, must undergo transformations in the process of resilience. Damage to the physical integrity of neural systems is present, but functions normally relying on these damaged resources appear preserved. It is reasonable as a first approximation to hypothesize that resilience is related to compensatory mechanisms that will be reflected in dissociations between normal structure-function relationships and pathological structure-function relationships for equivalent performance [34].

3.2. Evidence from Neuroimaging and Computational Modeling for Dissociations in Structure-Function Relationships and Renormalization of Function. Recent methodological advances in neuroimaging acquisition and data analysis have enabled the estimation in vivo of large-scale interactions in the brain, in normal and pathological contexts. Tractography in diffusion weighted imaging (DWI) can be used to quantify structural connectivity, and analyses of covariance in resting state functional MRI (rsfMRI) can be used to quantify functional connectivity. Advanced statistical analyses often based on graph theory can further be applied in order to characterize topological properties of structural and functional connectivity [36, 67, 81-84]. Studying the relationships between structural and functional connectivity is a suitable paradigm to investigate large-scale structurefunction relationships in the brain in connection to resilience.

Analyses of functional connectivity have shown that the brain is organized in functionally coherent large-scale networks at baseline (e.g., during wakeful rest and inactivity), the so-called "resting state networks" (RSNs) - that is, intrinsic networks constituted by sets of brain regions whose activity appears reliably correlated at rest [85]. Some of these socalled "resting state networks" (RSNs), such as the default mode network (DMN), have been associated with higherorder cognitive functions, including consciousness [86, 87], autobiographical memory [36], executive control [88], and language $[89,90]$.

Abnormal resting state functional connectivity has been implicated in multiple neurological disorders [91]. RSNs, including the DMN, were altered in patients diagnosed with $\mathrm{AD}$ [92-94] or at risk of $\mathrm{AD}$ [95], and in patients with traumatic brain injury $[96,97]$. Functional connectivity alterations appeared partially correlated with AD neuropathology [98]. DMN activity was correlated longitudinally with the course of AD $[66,94]$. Also, studies of RSNs in normal aging have shown age-related RSN changes $[99,100]$.

These networks appear to depend on complex mechanisms and can show flexible relationships to the underlying structural connectivity. Stationary neurodynamical models of physiological functions can predict, taking structural connectivity as an input, the topological and topographical organization of some of the main RSNs [101-103]. However, a large amount of prediction error variance between simulated and empirical functional connectivity remains and cannot be reduced based on the manipulation of structural parameters [102]. Nonstationary dynamics are likely to contribute to this variance. The relationship between functional connectivity and structural connectivity is complex and indirect (Figure 3).

Nevertheless, some systems of structural connections are predicted by a range of generative models to play a particularly critical role in shaping the overall topographical organization of RSNs across the brain. According to the models, the commissural system of homotopic fibers that passes through the corpus callosum and connects functionally homologous regions of the cerebral cortex across the two hemispheres plays a critical role in the emergence of bilateral symmetrical RSNs across the two hemispheres [102] (Figure 2).

However, a few studies of patients with corpus callosum abnormalities, including complete commissurotomy [104] and congenital callosal agenesis [105], have found RSNs to be remarkably normal and symmetrical in these patients. This challenges the idea that interhemispheric symmetries in major RSNs are necessarily mediated by callosal fibers and shows that functional networks can survive major changes in structural connectivity (Figure 3(a)).

Moreover, resting state fluctuations are large in magnitude and normally consistent across participants and 


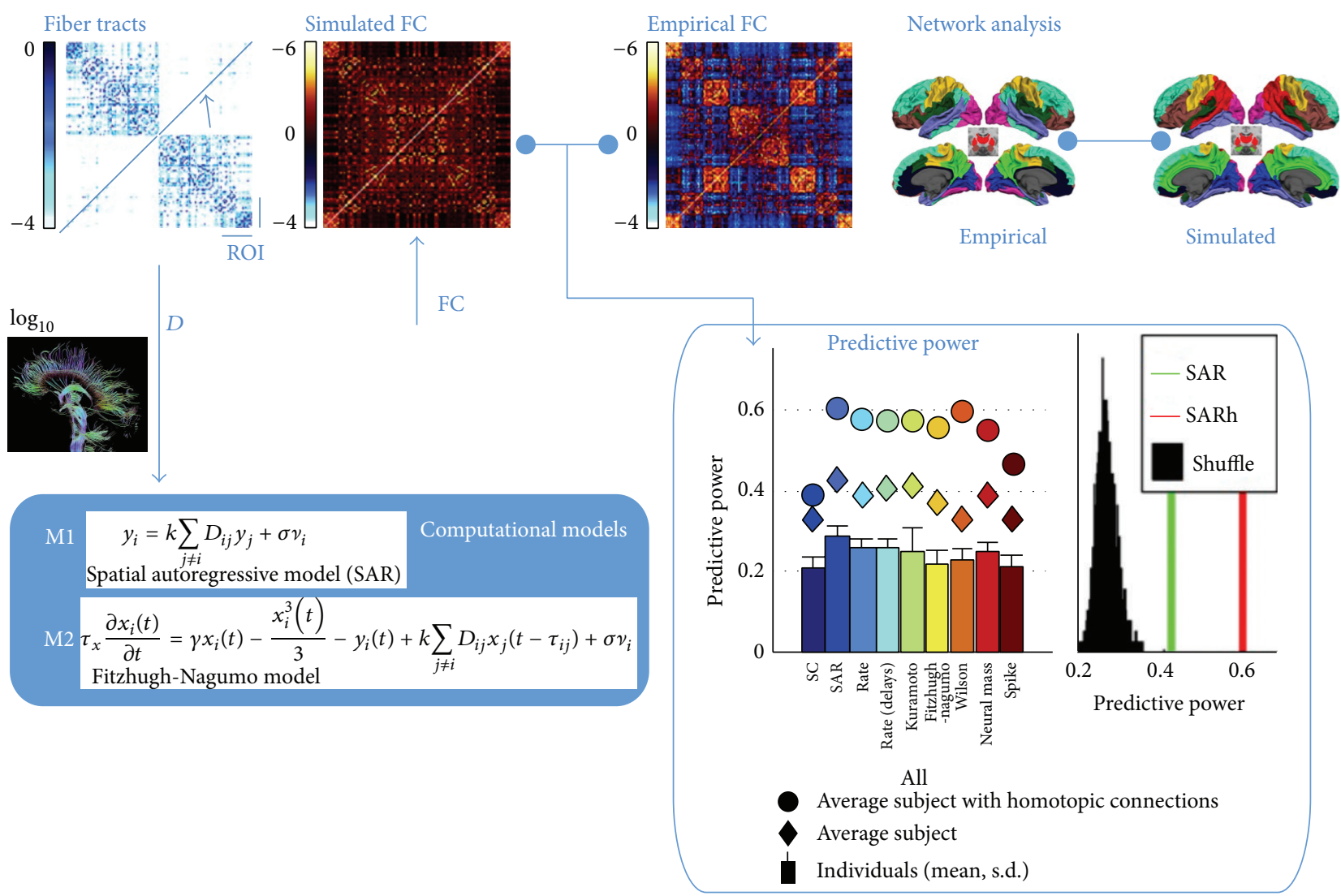

FIGURE 2: Modeling structure-function relationships. Left. Upper-tier: matrices of (anatomical, simulated-functional, and empiricalfunctional) average connectivity, between a set of 160 regions of interest derived from automated segmentations of the human cerebral cortex. Network analysis: connectivity information is also represented as cluster of similarly connected regions over the cerebral cortex for empirical and simulated functional connectivity (SAR model, i.e., spatial autoregressive model). Lower-tier: matrices of anatomical connectivity (from DWI-based tractography) are used as inputs to computational models of neurophysiology, with increasing complexity and realism (from SAR to Spike models), from which simulated matrices of functional connectivity are derived. Predictive power: the correlation between simulated and empirical data (predictive power) is highly increased (circle color dots) when missing interhemispheric and homotopic connections are added (the effect is specific to this fiber tract system and robust to permutation schemes [histogram chart]). (Adapted from [102]).

across time [106-109]. Part of these fluctuations might have functional significance and might be related to fluctuations in cognitive and behavioral performance. Fluctuations in functional connectivity following brain damage could be an important functional marker of the process of compensation and rescue of function, useful for the development of strategies of rehabilitation [110-112].

RSNs have a tendency to shift towards a more random structure of connectivity in patients with nonfocal brain pathology, for example, in brain tumors [113, 114], AD [93], epilepsy [115], and severe TBI [96]. A study of patients with multiple sclerosis showed that some RSNs were altered and others were reorganized [116]. There are a few studies in patients with more focal disorders. Carter et al. [82] studied 23 acute stroke patients and found that the dorsal attention network and arm somatomotor networks were altered. Moreover, the loss of coherence among different RSNs predicted behavioral deficits. Wang et al. [112] studied 10 patients with subcortical infarctions. They investigated RSNs across 5 consecutive time points within a single year and observed gradual changes in networks and associated functions. In particular, they found a tendency for the motor executive network to gradually transits through a random mode during the recovery process. Vanhaudenhuyse et al. [46] found that DMN connectivity was decreased in 14 severely brain damaged patients, in proportion to the patients' degree of impairment in consciousness (e.g., lockedin syndrome, coma, and vegetative state). Recent research on patients with disorders of consciousness, such as patients in a persistent vegetative state (PVS) or a minimally conscious state (MCS), also suggested the importance of RSNs' functional connectivity in functional preservation or recovery [4, 5, 42]. Nakamura et al. [96] demonstrated evidence of rapid renormalization of functional connectivity (3-6 months) in the process of recovery from trauma after an initial phase of high perturbation of the normal pattern of functional connectivity (Figure 3(b)).

In the framework of these large-scale structural and functional parameters, the problem of understanding and controlling the mechanisms of resilience in neurology is 

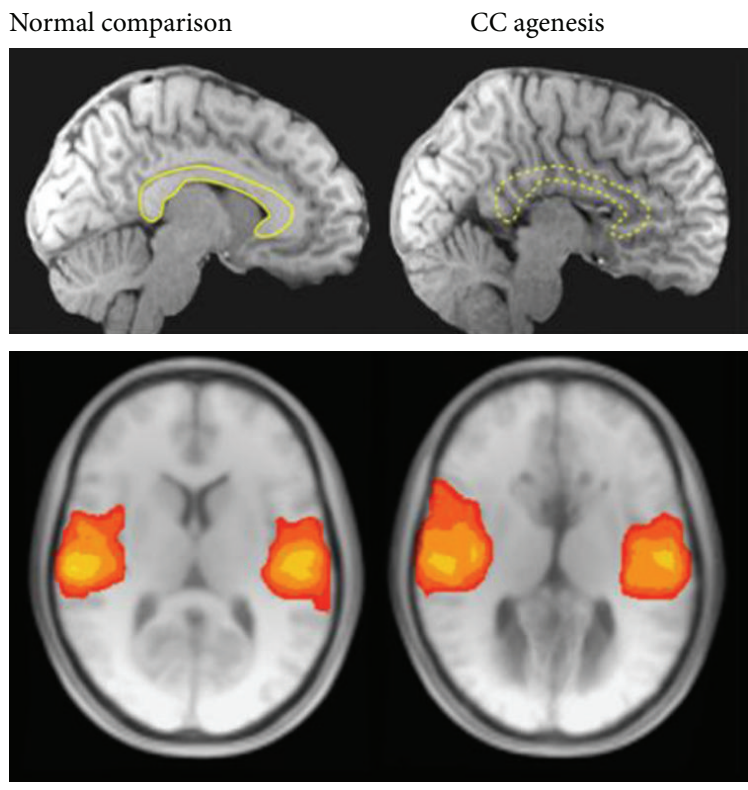

(A)

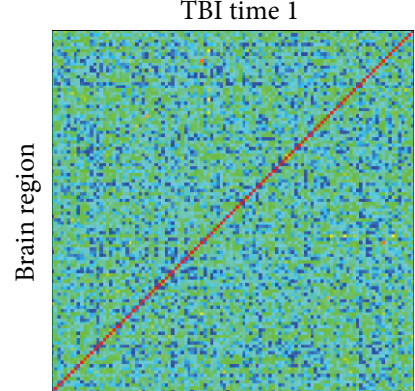

(a)

Control time 1

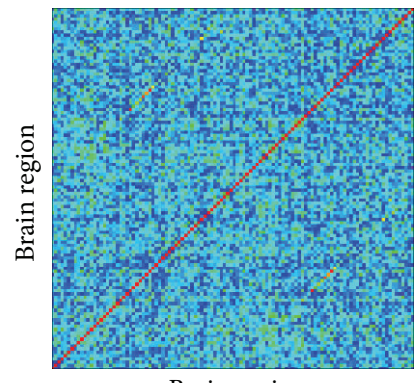

Brain region

(c)

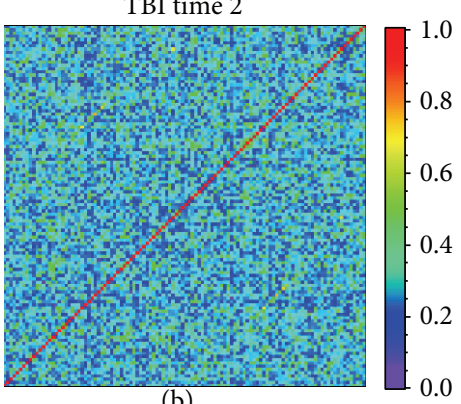

(b)

Control time 2

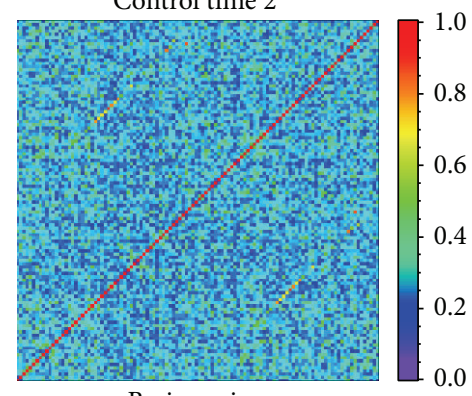

Brain region

(d)

(B)

FIGURE 3: Dissociations between functional and structural connectivity and renormalization of function. (a) Preserved bilateral, symmetrical functional connectivity from resting fMRI in patients with agenesis of the corpus callosum (from [105]). Top-tier: sagittal slices of T1-weighted MRI data from a normal comparison participant (left) and an agenesis patient (right). The normal location of the corpus callosum (CC) is indicated with dashed yellow lines. Bottom-tier: axial slices showing similar bilateral functional connectivity from resting fMRI in both patients and normal comparison participants. (b) Evidence for rapid renormalization of functional connectivity (3-6 months) in the process of recovery from traumatic brain injury (TBI) (from [96]). Charts represent matrices of functional connectivity derived from resting fMRI for the patients (upper-tier) and the comparison participants (lower-tier), at two time points after TBI onset (left versus right tiers). Functional connectivity in the patients progressively renormalizes in conjunction with behavioral recovery after an initial phase of perturbation.

a problem of modeling how normal functional connectivity can be preserved in the context of abnormal structural connectivity. Models can be modified to incorporate ad hoc neuropharmacological or electrophysiological mechanisms (e.g., additional norepinephrine-related neurotransmission; new inhibitory receptors-conductances; electrical potentiation of connectivity along alternate, indirect pathways) (Figure 4).

\subsection{Setting and Operationalizing Resilience: Brain Computa-} tion and Mutual Information. In order to better understand possible underlying mechanisms for resilience, it is useful to start from a simple operationalization of brain computation. Let us define the normal brain as a structural network $S_{N}$ of $n$ regions/nodes, associated with a generative model $G$ of the dynamics (generally a set of differential equations), yielding a functional network $M_{N}$, such that

$$
M_{N}=G\left(S_{N}\right)
$$

Both networks $S_{N}$ and $M_{N}$ can be described as weighted graphs associated with corresponding adjacency $n \times n$ matrices $S(i, j)$ and $M(i, j)$. An adjacency matrix is simply a square matrix informing the level of (structural or functional) connectivity between each pair of nodes.
Let us now assume that the brain performs some form of computation, executing processes $\{A, B, \ldots, Z\}$ which implement functions and operate over partially encapsulated (i.e., specialized) subsets of the brain network. One can conceive of such subsets or subnetworks as RSNs or task based networks (TBNs), that is, networks of brain regions that are reliably activated (e.g., in fMRI) during specific tasks [122]. Though they interact, such networks, which are related to different cognitive functions, can operate in a relatively independent manner (which is why they can be revealed with techniques such as independent component analysis for RSNs, and statistical contrasts between conditions for TBNs).

Within each of these subnetworks, network nodes interact through structural wiring to implement a function. The computation involves exchanges of information between the nodes. Here by node, we do not necessarily mean individual neurons, but also entire neuronal ensembles, which are partially specialized (e.g., cortical area V4 in the visual system) acting as a unit, depending on the scale considered (see Figure 5(a)).

We can further operationalize this model by describing the direct or indirect exchange of information between nodes using Information Theory and the concept of mutual information [123]. Mutual information is a quantity derived 


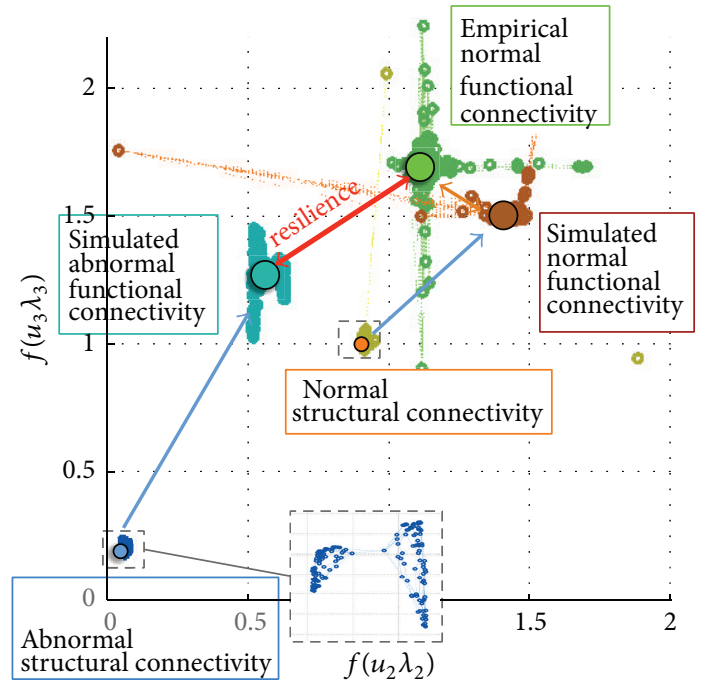

FIGURE 4: Research on resilience and modeling of structurefunction relationships. Example of Laplacian matrices of anatomical and functional connectivity, for empirical and simulated, normal and pathological instances represented in a vector space over two components following singular value decomposition and additional transformations providing an optimal layout and metrics for comparing topological properties of networks. A simple SAR model (see [102]) is used to predict matrices of functional connectivity from matrices of structural connectivity. Normal anatomical connectivity predicts a simulated-functional connectivity that is close to empirical-functional connectivity, whereas abnormal anatomical connectivity (simulated callosectomy) predicts abnormal functional connectivity, farther away from empirical-functional connectivity. The distance between simulated and empirical functional connectivity, given anatomical connectivity, provides the parameter of control to drive research on mechanisms of renormalization of function and resilience.

from Shannon's entropy [124], which characterizes the degree of statistical dependence between two variables $X$ and $Y$. It is minimal when two systems do not exchange information and maximum when they share the exact same information.

Let us now consider the case of a pathological brain with a damaged structural network $S_{P}$ and an abnormal functional network $M_{P}$. Let us say that two of the network nodes $(i, j)$ are now disconnected. This means that their effective connectivity $w(i, j)$, which is an indicator of presence of anatomical connectivity weighted by a transmission or synaptic gain (see Definitions), is equal to 0 . The nodes cannot directly interact and thus cannot exchange information. As a result their mutual information $m(i, j)$ is also equal to 0 .

However, if there are alternative, indirect pathways that structurally connect the nodes $i$ and $j$ with sufficient effective connectivity to provide bandwidth then the two nodes can interact and exchange information, and their mutual information $m(i, j)$ is not equal to 0 .

From the standpoint of resilience, it is also important to exploit alternative pathways that do not imply prohibitive transmission delays for the integrity of the cognitive process. It is customary to observe a slowing down of processing in brain damaged patients reflected in behavioral performance (e.g., [13]).

Likewise, if nodes or pathways become shared by different processes-for example, to compensate for lost resources pertaining to one of the processes by using alternative, indirect pathways associated with other processes-then access conflicts, cross-talks and interferences may arise between the processes, depending on how the transmission channels and effective connectivity are set. Phenomena such as tinnitus and pathologies such as epilepsy are pathological testimonies of such interferences.

Figure 5 presents simple illustrations of such an overall operational model from which we can analyze the implications of resilience for neuroscience.

\subsection{Redundancy: Structural, Topological, and Combinatorial} Bases of Resilience in the Brain. Redundancy is intrinsic to the brain's anatomofunctional architecture, and plays a natural role in its capacity for resilience. Redundancy may provide the brain with the ability to switch to alternate processing resources when others become unavailable or untrustworthy (Figure 5). From a combinatorial standpoint, the brain appears well equipped for functional resilience to structural challenges.

It has been proposed that the structural balance between segregation (functional specialization of subnetworks) and integration (their degree of interconnectedness), in the overall anatomofunctional organization of the brain presents a so-called small-world topology (see [125]). Such topology maximizes the number of short pathways between any two nodes in the entire network, while reducing wiring cost (overall number of necessary structural connections) and make the network's short connectedness resilient to disconnection. This hypothesis has recently been challenged (see [126]) to favor instead a bow tie architecture, in which dense peripheral subnetworks are highly pluripotent and not specialized. In both cases the architectures have properties of resilience.

More basically, structural parameters such as premorbid neuronal count and its volumetric proxies, the so-called "passive brain reserve" (see [8]), are highly relevant. The high number of neurons in the brain and between-subject differences in overall neuronal resources represents a strong source of redundancy and variability in redundancy. In principle, larger brains can sustain more insult up to a certain threshold.

However, redundancy in the brain is intimately related to functional parameters. It is expressed in at least two complementary ways (Figure 5(d)) [2, 14, 127]. There can be a relative "degeneracy" among processing units/nodes: different nodes are capable of handling the same function or output, it is a many-to-one mapping (e.g., parallel array of identical specialized processors). There can also be a relative "pluripotency" in processing units: individual nodes are capable of handling multiple functions contextually; it is a one-to-many mapping (e.g., processor individually executing different tasks sequentially by switching between them). Examples of possible anatomical substrates for such processes would include, with unilateral lesions, contralateral homologue regions taking over; the direct penumbra of the lesion, 


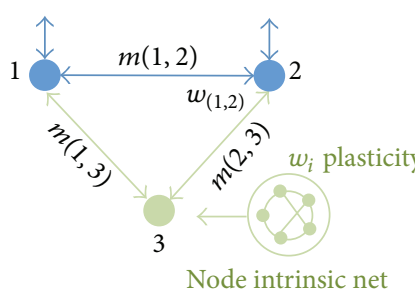

(a)

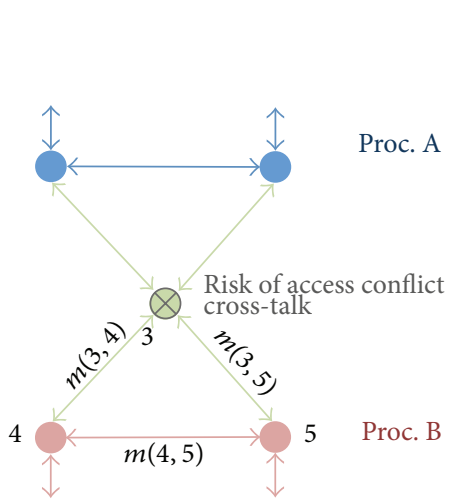

(b)

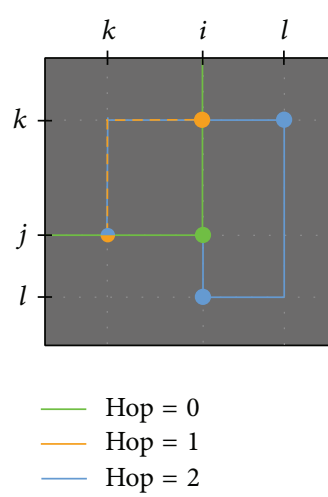

(c)

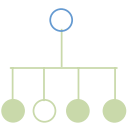

Degeneracy

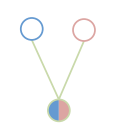

Pluripotency

(d)

Figure 5: Redundancy and resilience. (a) Simple model of network with 3 nodes (1, 2, and 3). Nodes 1 and 2 interact to perform process A (blue), implementing a function, with inputs and outputs from and to other unrepresented nodes (little blue arrows). Node 3 is structurally connected with nodes 1 and 2 but is assumed to present little effective connectivity $w(i, j)$ and thus not to interact much with nodes 1 and 2 . Each node, which can be a region of the brain, can contain multiple intrinsic processing units (subregions or neurons), working together and capable of plasticity, that is, of modification of their effective connectivity via synaptic tuning. One can define a quantity of mutual information $m(i, j)$ between any two nodes $(i, j)$ in the network (see text). If the effective connectivity of node 3 with nodes 1 and 2 increases, node 3 will relay information between nodes 1 and 2 , and the chaining of $m(1,3)$ and $m(2,3)$ will contribute to $m(1,2)$ : node 3 and its wires can become an indirect, parallel, alternate carrier of signal for nodes 1 and 2. This represents a form of redundancy. (b) Extended model from (a) with two additional nodes, 4 and 5, assumed to support a process B, irrelatively independent from process A, through their interactions. A quantity of mutual information can also be defined between nodes 4 and 5, and any other nodes in the network. In this case, node 3 is placed in a position of "hub"; that is, it is highly connected structurally with the other nodes of the network ("degree" $=4$ versus 2 connections). If its effective connectivity with all its neighbors (i.e., directly connected nodes) is increased, it will start indirectly relaying signals not only among nodes of the same process (A or B), but also between nodes subserving the different processes (A and B). Without further filtering to separate and channel sources of signals, there is thus a risk of cross-talk and interferences between the processes $\mathrm{A}$ and $\mathrm{B}$. If for functional reasons, the processing capacity of node 3 becomes shared by processes $\mathrm{A}$ and $\mathrm{B}$, and plasticity within the node manages to reduce crosstalk between them, for example, by serializing the access, and to handle the source separation and channeling problem, there is nevertheless a risk of "access conflict" for the resource represented by node 3. (c) Theoretical matrix of structural connectivity (i.e., adjacency matrix) informing the existence of structural connections between each pair of nodes in a network. The analysis of such matrix can identify all direct and indirect, alternate structural pathways between any two nodes $(i, j)$, and sort them according to their "hop" distance, that is, the number of intermediary nodes along the pathway connecting $i$ and $j$ (which is 0 when the connection is direct, 1 when it has one relay, 2 when 2 , and so on). (d) Two types of functionally relevant redundancy: degeneracy and pluripotency (see text). Assuming that it is functional, node 3 in (b) can potentially represent a pluripotent resource for both processes A and B.

that is, tissues around the lesion, being sufficient to compensate; or entirely different structures and circuits, not already pluripotent, being recruited to compensate. More generally, individual neurons are capable of switching between different information processing modes in a pluripotent and highly contextual manner, notably in the prefrontal cortex (see [128]).

Further mechanisms have been discussed in the field, often in the context of the notion of "cognitive reserve" [8, $11,72,129]$. Resilience can build upon preexisting cognitive skills and thereby facilitate problem-solving in cognitively challenging environments. It can use resources within brain networks that normally support these specific cognitive skills, or alternate resources that do not normally support such skills. It can optimize the balance between two main categories of parameters. The first parameter is "efficiency," minimizing the number of operations and energy expenditure. The second is "capacity," that is, the maximal processing power or capacity of integration per neuronal unit, based on which a stronger processing demand can be placed on preserved neurons by making them operate at fuller capacity.
It is generally the case that brain damaged patients demonstrate slower cognition and behavior, often accompanied with decreased cerebral metabolism such as in AD. This suggests that both capacity and efficiency in their brain reach a limit. In $\mathrm{AD}$ (see above), patterns of higher metabolic activity in certain regions of the brain have been hypothesized to reflect compensatory mechanisms [66]. This could be an illustration of a case of increased processing capacity per unit time for neuronal units in these regions. Activation studies using fMRI can study efficiency and capacity in brain processing using event-related designs in which cognitive load during task is manipulated [8].

Alternatively, the brain can build upon a flexible use of cognitive strategies and brain systems that are not normally involved in the skills (e.g., mnemonic strategies to compensate impaired recall, and interhemispheric transfer of psycholinguistic functions after a stroke). Partial evidence suggests both an enhancement of the processing in neural networks that are specifically related to the task demand, and the participation of more general "cognitive reserve networks," involving frontal sectors, which have been otherwise 
associated with controlled processes, task switching, and working memory $[66,72,79]$. Emotional and motivational factors may also contribute to fueling resilience [130], notably via the noradrenergic tone [131].

Thus cognitive resilience can rely on the combinatorial power of redundancy (degeneracy and pluripotency) in the anatomofunctional architecture of the brain, including on the presence of multiple alternate indirect pathways [2, 13, 22]. It can build upon a plastic capacity for adaptively switching between alternate resources, as well as upon mechanisms optimizing efficiency of processing within networks.

\subsection{Renormalization of Function, Dynamic ReRouting, and} Plasticity: A Complex Inverse Problem over the Structural Backbone for the Brain. Under the models described above, resilience corresponds to a general "renormalization" of the core functional/processing architecture supporting cognition (e.g., as suggested in $[96,105]$, in the context of analyses of functional connectivity from fMRI; see Figure 3). Such renormalization occurs in the context of a severely modified processing and wiring structure. By "renormalization" I explicitly mean a relative preservation of - or return to-the "normal," overall core computational scheme characterizing normal cognition. This process must be achieved in a manner that is compatible with available structural and metabolic resources, given a structurally modified brain network and a corresponding loss of previously dedicated processing power. This does not imply that the overall computational scheme of cognition is not substantially modified in its details. Multiple strategies may arise for specific cognitive processes.

For such renormalization, the brain must solve a routing problem. It must be capable of managing and recombining resource allocation adaptively via the dynamic "rerouting" and addressing of distributed signal streams along alternate neural pathways at multiple scales. It must dynamically identify, address, and allocate redundant, functionally compatible residual resources by a two-pronged approach: bringing alternate neural resources online, while coping with other neural resources that are dysfunctional and thus untrustworthy (e.g., noise in sensorimotor systems related to disease processes), for instance by setting them offline in order to minimize perturbation (e.g., progressive inhibition). The frequent observation in many conditions [1], as it was also the case for the patient Roger [3], of strikingly rapid recovery from extensive damage that had initially caused profound cognitive deficits (e.g., stupor, delirium, neglect, and anosognosia), emphasizing the dynamic and massive nature of such reboot and general reorganization.

As damage becomes more extended (e.g., in the course of $\mathrm{AD}$ ), the brain must face increasing difficulties in finding resources available for compensation, in order to preserve core, critical cognitive functions. Solving the allocation and routing problem underlying resilience must become increasingly difficult. It is predictable that the brain will encounter more and more access conflicts for resources (Figure 5) and will have to rely on the pluripotency of residual resources. Such an increasing reliance on pluripotency can only result in slower, noisier, and ultimately dedifferentiated processing, with a lower capacity for information processing. This implies subsequently placing more stress on available resources, as a condition for maintaining relative efficiency and specificity in a manner that is compatible with the timing and energetics of core adaptation. Such suboptimal processing can eventually become incompatible with the throughput required for adaptation and functional coherence. To some extent, this process is somewhat reflected in resilient $\mathrm{AD}$ patients who generally reach an inflexion point in their mental status. At this point, the cognitive apparatus that had been, until then, relatively resilient (generally at the expenditure of a high metabolic pressure over remaining functional tissues) collapses dramatically, plunging the patients into a rapidly progressing dementia. Such inflection point is observed at stages that are closer to organ failure in resilient patients than in nonresilient patients [8].

The overall guiding principles of the execution of such renormalization and the control of this global reweighting of effective connectivity in the brain remain mysterious.

The capacity for renormalization of function must at least in part be self-contained in the brain and robust: it operates and deploys in a rather systematic manner, at the cost of much energy expenditure, since all living individuals are more or less resilient, in a more or less efficient and powerful manner, as it is obvious in the psychological struggle of humanity [17]. It must itself be one of the most resilient general features of brain computation: a process in charge of controlling overall resilience in the brain must be itself resilient to fulfill its function.

The brain can functionally reconnect itself after damage via alternate, indirect pathways, based on local mechanisms of plasticity related to learning and adaptation, such as the modulation of synaptic plasticity [80]. Modulating synaptic efficacy, that is, synaptic transmission gain, which is equivalent to modulating effective connectivity in the network, will control information transfer and can be used to restore it (Figure 5). Figure 6 illustrates these concepts using the simple model of brain processing introduced above (see Figure 5), by showing how structural connectivity, effective connectivity and mutual information interact in a small network undergoing disconnection and synaptic plasticity over pluripotent resources. After disconnection of two nodes performing a coordinated process in the model, information flow is disrupted. The process fails. It can be partially restored, as reflected in their mutual information, thanks to a reweighting of the effective connectivity matrix, enabling indirect pathways to carry signals between the two disconnected nodes.

Yet, this process of synaptic plasticity must operate over massively distributed local nodes. Deciphering how such rerouting scheme can be coded and deployed in the brain is key to understanding fundamental relationships between structure and function in neuroscience.

As a first approximation and in all generality, the problem can be formulated as an inverse problem (see [132]). In our reduced model, the forward problem corresponds to that of defining a generative model $G$, taking a matrix of effective connectivity $W$ as an input and outputting a matrix of mutual information: $M=G(W, S)$, with $S$ the associated binary matrix of structural connections. The inverse problem 


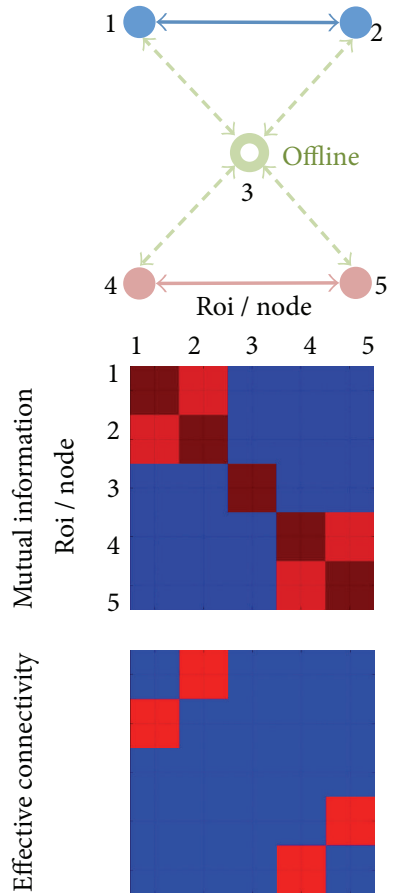

(a)
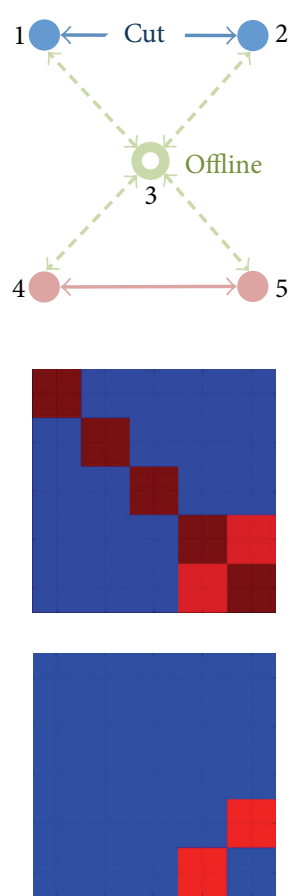

(b)
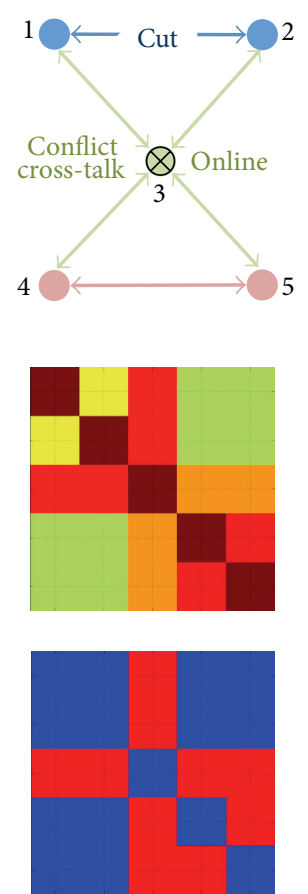

(c)
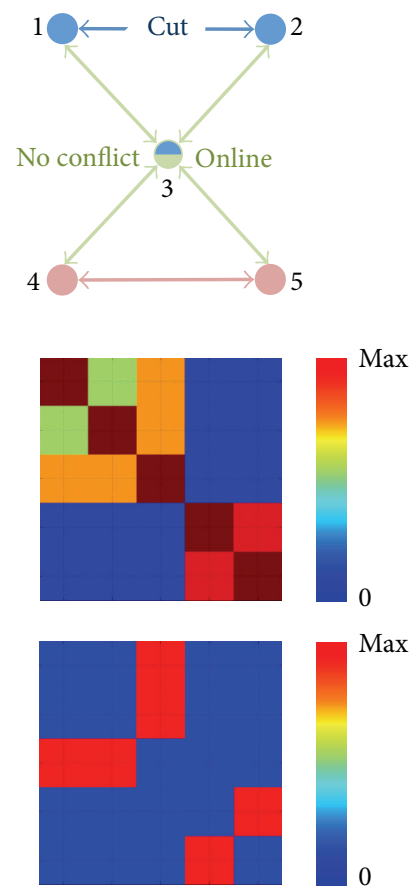

(d)

FIGURE 6: Information transfer and functional resilience in a model of small network subjected to disconnection. The principles, basic nodes, and (two-way) connections of the model are the same as in Figure 5(b). Upper-tier. Graphs of different states of the basic model. Two processes A and B implement some functions within, respectively, nodes $(1,2)$ and nodes $(4,5)$. It is assumed that the processes rely on an exchange of information between their respective nodes to compute the output of the functions. Disruption of information flow between nodes after structural disconnection (b, c, d) implies a failure of the process. A central node 3 or "hub" is connected to all the other nodes. Node 3 can be "offline" (a,b); that is, its effective connectivity with the other nodes is very low, and thus signals cannot flow through it even in the presence of structural connections. Alternatively it can be "online" (c, d); that is, its effective connectivity with the other nodes is high, and thus signals can directly flow through it. When node 3 is online, cross-talks and interferences between processes will arise (c), except if the node internal structure fully supports pluripotency (d), in case that information flow between nodes 1 and 2 is restored indirectly via node 3 without interference. Lower-Tier. Adjacency matrices of effective connectivity (bottom row) and associated mutual information (top row) for the different states of the network in ( $a, b, c$, and d). Each square within the matrix represents the strength of the corresponding parameter between two nodes $(i, j)$ among the 5 nodes of the network. The color bars on the right indicate the relative strength of the parameters. Model specification and simulations. In order to demonstrate the property illustrated in (a, b, c, and d), we used a simple generative model. Binary matrices of structural connectivity were first defined to implement the structure of connectivity. Matrices of effective connectivity were defined by reweighting the matrix of structural connectivity in order to implement the different states of effective connectivity in the network in (a), (b), (c), and (d) (e.g., high versus low connectivity; disconnection). Effective connectivity matrices were used as an input to a spatial autoregressive model (SAR), which demonstrates the best predictive power among many other more complex and realistic models in predicting empirical functional connectivity (from fRMI), based on empirical structural connectivity (from DWI) (see [102]). The SAR model assumes a stationary, multivariate, independently, and identically distributed Gaussian process with covariance matrix $F$. It corresponds to the equilibrium solution of a simple linear dynamical system of $n$ nodes interacting through a set of structural connections, with effective connectivity matrix $W$. The covariance matrix has a closed form solution with the SAR model and can be computed based on the matrix of effective connectivity $W: F=s^{2}(I-k W)^{-1}(I-k W)^{-1 T}$, with " $s$ " being the variance of the process, " $I$ " being the identity matrix, $(\cdot)^{-1}$ and $(\cdot)^{T}$, respectively, being the inverse and transpose of the matrices within the parenthesis, and $k$ being a parameter of overall connectivity within the network (here $k$ was set to .8 ). The covariance $F$ corresponds to what is measured as functional connectivity in functional neuroimaging: functional connectivity is generally defined as the covariance between measured signals. A correlation matrix $R$ can be derived from the covariance matrix $F$, and there is a simple relation between the correlation $r(i, j)$ in the dynamics of two nodes $i$ and $j$ and their mutual information (expressed in bits of information) because of the statistical properties of the SAR model. Thus $m(i, j)=\ln \left(1 / \operatorname{sqrt}\left(1-r(i, j)^{2}\right)\right.$ ), with $\ln (\cdot)$ being the natural logarithm and sqrt( $(\cdot)$ being the square root functions. (a) Information flows between nodes 1 and 2 , thanks to their high effective connectivity; mutual information is high between them. (b) Nodes 1 and 2 have been structurally disconnected, information does not flow, and their mutual information is zero. (c) Nodes 1 and 2 have been structurally disconnected, information does not flow directly, but node 3 has been brought online and information can flow through it indirectly. Mutual information between nodes 1 and 2 is partially restored, but there are also interfering cross-talks between nodes $(1,2)$ and $(4,5)$ through node 3 (see green squares in the corresponding matrix). (d) Nodes 1 and 2 have been structurally disconnected, information does not flow directly, and node 3 has been brought online but in a pluripotent manner that filters out cross-talks, resulting in no mutual information between nodes $(1,2)$ and $(4,5)$. Mutual information between nodes 1 and 2 is partially restored, as signals can flow through node 3 . Information processing has been partially renormalized by playing on effective connectivity over redundancies in the model. 
consists in finding an inverse generative model or inverse map $G^{-1}$ to infer $W$ from $M$ and $S: W=G^{-1}(M, S) . G^{-1}$ must select the set of $W_{i}$ that are compatible with $S$ and the topology of its embedded pathways. The problem of the brain then becomes a problem of optimization, which can be formulated as a process of minimization of the difference between a target, internal model $M_{N}$ of normal information processing, and an internal measure of pathological information processing $M_{P}$, such that the renormalized matrix of effective connectivity $W_{R}$ is chosen as

$$
W_{R}=\operatorname{argmin}\left\{\left|M_{N}-M\left(W, S_{P}\right)\right|\right\} .
$$

$M\left(W, S_{P}\right)$ corresponds to the predicted measure of information processing $M$, given a matrix of effective connectivity $W$ and the pathological structural connectivity matrix $S_{P}$, assuming an internal forward model: $M=G(W, S) . W_{R}$ is the matrix $W$ that minimizes the difference between normal information processing and a possible alternate implementation of information processing given the constraints imposed by $S_{P}$. Internal measures of the discrepancy between the current pathological matrix of effective connectivity $W_{P}$ and the normal internal model $W_{N}$ associated with $M_{N}$ can then provide signals to control local synaptic plasticity in order to renormalize function.

In this framework, in order to be resilient, a brain must therefore embed fairly abstract, innate or acquired, internal, normative models of itself and of processing strategies serving as a compass for adaptive cognition and renormalization. It must also embed an algorithm that can perform such minimization, in a manner that is energetically and functionally viable and robust. One option that could be envisioned as a possible solution to the global renormalization problem would be to implement processes similar to simulated annealing and random walk search. They might account for the between-subject variability observed for similar cases of damage in recovery of function, both in terms of timing and performance. It is likely that what matters is to optimize the output function of processes, that is, optimize functions in a task-oriented manner based on desired goals that are internally modeled and a signal of reinforcement. This is compatible with textbook knowledge about operant learning in the brain.

3.6. Local Synaptic Plasticity and Resolution of Conflict in Pluripotent Nodes. The hypothesis is that, at the lowest level of implementation, all local computational principles and corresponding biophysical and biochemical mechanisms involved in synaptic plasticity and learning, that is, one of the key interface between structure and function, are exploited by such an overall algorithm of renormalization to tune the routing of signals and transfer functions between and within neural ensembles, that is, in this framework, effective connectivity.

One of the problems that local synaptic plasticity will have to resolve is the mutualisation of a limited pool of neural resources to reconnect disconnected systems and replace damaged ones. It will have to create well-tuned properties of pluripotency in neural resources as they become shared between different processes, for example, for providing an alternate, indirect channel of information transfer between disconnected nodes associated with the different processes (Figures 5 and 6). Cross-talks between processes can occur during renormalization as access-demand by the different processes for the shared bandwidth of the pluripotent node increases the risk of conflicts and interferences (Figures 5 and 6).

In principle, synaptic plasticity can drive intrinsic units in pluripotent nodes to channel processes by dividing their total bandwidth for different client processes. This may be done either in a serial manner, for example, by enabling switching between processes over time through cross-inhibition, or in a parallel manner, for example, by frequency filtering/multiplexing or the local rewiring of resource into dedicated, parallel physical pipelines. In all case, it generally involves resolving a problem of blind-source separation [118]. Figure 7 illustrates such mechanisms. Blind-source separation can be performed by neural network implementations of standard statistical algorithms, based on various versions of classical leaning rules such as the famous Hebb rule (see [119]; Figure 7), which possess some degree of biological plausibility, supported by empirical evidence (e.g., NMDAreceptor-mediated long term potentiation).

But, even though, the implementation of such algorithms in artificial neural networks is relatively straightforward [121], there are many customary issues concerning the normalization and stabilization of the underlying process of reweighting of effective connectivity/synaptic gain over the ensembles of units in the network [133]. For many neural network implementations of these algorithms, it is necessary, in order to avoid divergence and enable convergence, to perform for each iteration of the learning process a normalization of the local synaptic weights in the network, that is, of effective connectivity. This must be done based on a measure of the overall weight of connection in the entire network. Such constraint somewhat defeats the purpose of providing implementations of learning algorithms based on purely local plasticity and principles. In such cases, individual synapses must still "know" the states of all the other synapses to renormalize their own weight. This comes down to expecting neurons to encode routing tables of the entire network or to broadcast information about their own effective connectivity to others or to have access to a central repository or hub capable of informing the entire network about its own state by broadcasting global parameters (see [134]). Evidence indicates that sleep and dreaming may be key periods during which brain processing enters a special mode in order to perform memory encoding and globally normalize local synaptic weights after a day of overall synaptic potentiation related to active, wakeful learning [135].

3.7. Resilience: The Manifestation of Layers of Abstraction at the Core of Structure-Function Relationships in the Brain. In resilience, the renormalization process involves something global, and the relationships between the "global" level (general routing scheme and strategy) and the "local" levels (synaptic plasticity) appear and remain quite mysterious (see 


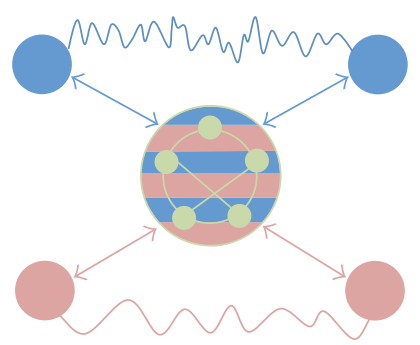

(a)
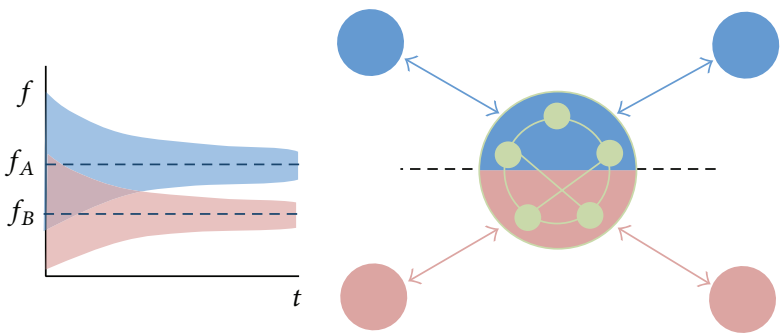

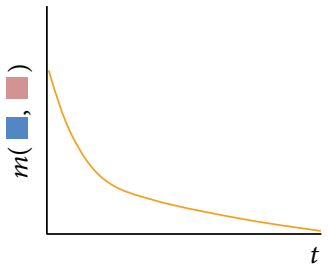

(b)

FIGURE 7: Plasticity and pluripotency. Pluripotent nodes can be recruited to provide a common alternate relay between groups of nodes subserving different, independent processes (Figure 5). As the different processes compete for the pluripotent node's bandwidth, the pluripotent node's intrinsic subnetwork of processing units must learn to carry and output the signals from the different competing processes in a manner that is ultimately unadulterated. One option is to multiplex signals from different processes over the subnetwork, for instance based on their frequency content (a). If two processes $A$ and $B$ (blue and red) generate nonoverlapping, narrow-band signals with different central frequencies $f_{A}$ and $f_{B}$, the subnetwork can learn to tune its output nodes' transfer functions so that they separately filter the two frequency bands, and relay adequately the signals (see little chart on the right). Oscillations and the emission of power from multiple frequency bands over the same regions of the brain are reliably observed in electrophysiological signals (see [117]). Another option is to keep the signals from the different processes separated within the subnetwork over different, potentially cross-inhibiting subpipelines. Many other options can be envisioned. In all cases, somewhat, the pluripotent node must learn, as it is recruited for renormalization of information transfer, to separate independent sources of signals (b). Blind source separation can be performed by unsupervised algorithms such as principal component analyses (PCA), for uncorrelated sources of Gaussian signals, and by independent component analysis (ICA), for statistically independent sources of signals (see [118]). In both cases, source separation enables the absence of cross-talks and interference between processes. This is expressed in a mutual information $m(i, j)$ between the nodes of different processes that becomes null (see (b) little chart). These algorithms can easily be implemented in small neural networks. PCA is formally equivalent to neural network implementations based on classical learning rules from connectionism such as Hebb's rule for unsupervised learning (see [119]). Hebb's rule increases synaptic weight $w(i, j)$, that is, local effective connectivity, between a presynaptic neuron $i$ and a postsynaptic neuron $j$ based on the presence of sustained correlations in their activity (see [120]). In this framework, synaptic weights behave just like the loadings of principal components along directions of maximal covariance. ICA can be implemented with modifications and generalizations of Hebb's rule in a manner that minimizes mutual information between processes while maximizing their own intrinsic quantity of information (e.g., infomax algorithm, [121]).

[136]). In order to approach this issue, we must first wonder what processes are the main targets for renormalization in resilience.

The brain is a highly complex biological machine that resolves extremely difficult problems of coordination, learning, inference, anticipation, modeling, integration of information, constitution, and storage of knowledge, as well as sequencing and execution of commands and actions. It does so through a unified framework or "global workspace," for perception, imagination, learning, inference, decision, and action that we call our minds when we are awake (see [127, 137-140]). At the core of this framework there is what I have called "higher-order cognition," which encompasses and unites together in the subjective frame, executive functions, attention, general intelligence, and self-awareness; which are all functionally related. For instance, in healthy individuals, higher metacognitive skills are associated with greater intelligence [141]. Dementia corresponds to a dramatic and radical dissolution of such integrative cognition.

As will be explained in detail in future reports I hold that such a cognitive framework of the human mind is characterized by two main components, for which we recently gained indirect empirical evidence [122]. On the one hand, there is a central process of ongoing computation of inferences in frontotemporal cortices on the physical and social causes of sense data, based on internal models of the world and self, and heavily relying on language and symbolic logic (following principles similar to a hierarchical Bayesian scheme). This process heavily relies on long-term memory and operates over time to satisfy a core utility function related to innate or vital goals and needs, which is expressed through emotion and feeling. On the other hand, there is the real-time multisensory computation and rendering in posterior cortices of a specific type of projective spaces (in a technical sense of that term in projective geometry; see [142]), which frames our three-dimensional experience of the world and self, in perception and imagination, and more generally in spatial cognition. Such internal projective space also serves for the programming of actions in connection with the real space to which it is coupled via sensorimotor interfaces. These two central components of cognition are functionally coupled, just like thought and perception or emotion and action are in the vocabulary of classical philosophy. Their anatomical substrate may be reflected in major anatomofunctional subdivisions in the brain recently uncovered by our group [122].

Irrespective of the details of the mind's cognitive framework, one can safely postulate that such framework exists and directly depends on the overall functional scheme of information processing in the brain. From a neurological standpoint, this functional scheme thus appears most important to preserve for overall cognitive resilience. It is first and foremost at the level of this "functional brain," so to speak, that the brain as a whole must be resilient to preserve cognition. 
Neuropsychiatric conditions such as schizophrenia, and their wealth of distress and anxiety for the patients, which is notably related to their frequent self-awareness of global cognitive dysfunction, or autism are a striking demonstration of the importance of preserving the overall cognitive framework, as a condition for autonomy and adaptation to the world's demands [17].

A key fact to integrate is that part of the process underlying resilience is driven by top-down cognition. Often, in the process of recovery, patients painstakingly struggle to rebuild awareness of their deficit, as a first necessary step for learning solutions to cope with it. Awakening and recovering from coma, vegetative states and minimally conscious states to regain full-fledged consciousness are striking examples of such struggle. They are cruel evidence that rebooting a general state of consciousness is the first critical step in cognitive resilience, before any other recovery process pertaining to more specialized skills can be engaged willfully to regain autonomy (see [43]). Then becoming self-aware of deficit, as disorienting and distressing as it may be, is, in and of itself, a root principle for reorganization: one must become aware of malfunctioning to be able to willfully act upon any malfunction. Anosognosia, the lack of self-awareness of one's own deficits, is negatively correlated with resilience [78]. There is clinical evidence that in many cases, higher-order cognition, for example, self-awareness, plays a key role in enabling and driving cognitive resilience itself. The concept of CR discussed previously brings forth the importance for resilience of neuropsychological variables such as general intelligence, degree of literacy, educational attainment, occupational complexity, interpersonal skills, integration in social networks, personality variables, and leisure activity.

More generally, self-representations, self-awareness of deficits, executive functions for self-control, goal-directed cognition, and intelligence, all, are enabling conditions and motivating factors for engaging in a process of frustrating, often painful and uncertain, sometimes lifelong rehabilitation.

In such context, if resilience implies a global reweighting of local synaptic plasticity, then there is a top-down control mechanism based on seeds of preserved higher-order cognition that is capable of acting upon local plasticity to support resilience.

Moreover, it is impossible to overlook the importance of social cognition and support in such process [143]. A supportive social environment of clinicians, friends, and family, providing social feedback signals can be key for resilience. It is a source of normative models of cognition and behavior, which can potentially be used by the patient in order to define cognitive and behavioral references or standards and better supervise the renormalization process as he struggles to find the pathways of resilience. This principle is at the center of most rehabilitation strategies.

At a slightly lower level of cognitive integration, phenomena such as sensory substitution (see [144]) demonstrate that, at an intermediate level, basic perceptual experiences are synthetized based on flexible relations to the brain hardware. In sensory substitution, a category of experience (e.g., visual) is induced in a functional manner, based on the stimulation of sensory systems normally associated with other categories of experience (e.g., touch). The stimulation is designed to reproduce sensorimotor contingencies that are intrinsic to the first category of experience (e.g., telemetry versus direct contact), based on intermediary devices augmenting the sensory channel associated with the second category of experience. The ability of the brain to sense the same sources of information and to build the same overall qualitative experience in multiple indirect ways is also illustrated by patients without insula who can nevertheless continue feeling interoceptive information via alternate pathways normally associated with exteroception [13].

Therefore, a hierarchy of top-down processes contributes to controlling resilience and renormalization of effective connectivity in a robust manner, from higher-order selfawareness and perceptual gestalt synthesis to basic operant learning.

In order to understand in an operational manner what exactly must be renormalized in resilience, it is important to identify specific levels of expression of cognitive processing that are as close as possible to observable physical levels of implementation. Information processing in the brain must ultimately embed cognition, even when considered at a level of observation close to hardware implementation (see the illustrative models presented in Figures 5-7).

At such level of implementation, information processing, for example, mutual information, at first appears to entertain a close relationship with the structural network, for example, matrices of structural and functional connectivity resemble each other and there are generative rules between them. However, as reviewed above, evidence suggests that the renormalization of functional connectivity correlates more with recovery of function than the rigid structure of processing units and wires that remains largely invariant at the largescale, following extensive brain damage.

The effective, in-use, functional wiring diagram of information processing supporting ongoing core cognition in the brain is to some extent different from the structural implementations that may be expected to serve as a preset, somewhat rigid, specialized set of pipelines to support it. As reviewed in this paper, the robustness of higher-order cognitive functions and their striking ability for resilience demonstrate that central aspects of higher-order cognition may rely on information processing mechanisms that are unlikely to rigidly depend upon a static, fixed set of specialized brain regions and anatomical connections. They are more likely to operate by dynamically exploiting available neural resources and possible routes between them, based on the resilient use of flexible, degenerate, and pluripotent brain systems. If higher-order cognition is so transferable and capable of dynamic reinstantiation and reinitialization, then, in principle, higher-order cognition might have multiple solutions of implementation with respect to the brain substrate [2]. More generally, in normal conditions, neural networks show collective behaviors that are manifested in higher-order parameters controlling highly contextual information processing modes, notably in the prefrontal cortex (see [128]). 


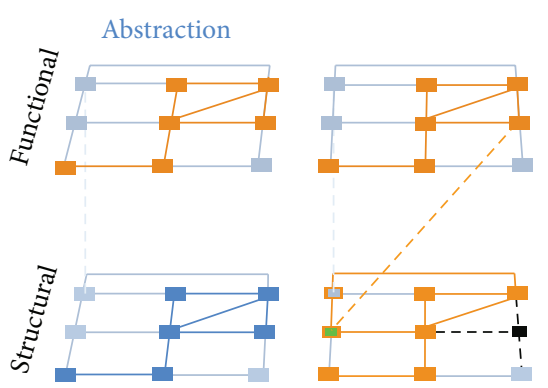

(a)

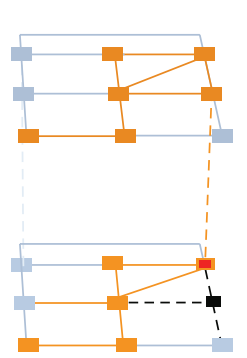

!

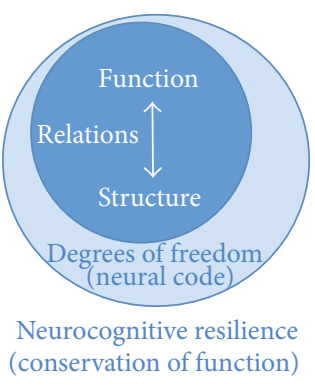

(b)

FIGURE 8: Abstraction of function from structure. (a) The relationships of connectivity among the nodes of a network can reside at, at least, two levels, structural and functional levels. Modifications at the structural level (black squares and lines representing damage) can be compensated by reassigning nodes and nodes' relationships to keep invariant (or similar) the map of the process of information transfer at the level of the functional map. Left. The structural and functional map over the network are identical. Center. After damage, the structural map is modified. Degeneracy in the structure is used to compensate for the lost node (black) and another available node in the structure is allocated (green). Topologically, the functional network is equivalent to the original structural network. Right. Pluripotency is used to compensate for the lost node, while another node, but already used in the structure is allocated (red). This nevertheless maximally preserves network's topology. (b) Illustration of the notion of degrees of freedom in structure-function relationships in the brain, and of their role in resilience.

Such capacity for relative decoupling between structure and function in the brain appears quite adaptive vis-àvis evolution. It implements a somewhat universal resilient functional architecture capable of rerouting and sustaining itself, in a manner that is partially independent from the fixed structure of connectivity that is most directly susceptible to irreversible damage. In summary, it suggests the hypothesis that

(i) core higher-order cognition behaves like a general algorithm that relates to the brain qua a hierarchy of layers of abstraction, and participates in driving its own resilience in a top-down manner (qua selfawareness);

(ii) interfaces between layers exploit combinatorial principles, just as with code execution in computers, in order to handle resource allocation and dynamic routing,

(iii) the process ultimately does so by dynamically controlling local biophysical and biochemical parameters involved in synaptic plasticity.

In other words, under such hypothesis, the relation between higher-order cognition, the brain, and the principles driving resilience appears to be of a relatively abstract or algorithmic nature. In this framework the neural correlates of the mind has to be understood via layers of abstractions (Figures 8 and 9). Resilience would reflect the presence of intrinsic degrees of freedom in structure-function relationships. It would be linked to combinatory rules implemented in the brain for flexible allocation of resources, operating at the interface between structure and function. The rules and mechanisms of these flexible relationships, which are nothing but the neural "code" itself, need to be studied, understood, modeled, and exploited in medicine and neuroengineering. I would even argue that there is no definitive reason at this point to reject the hypothesis that such code may exist and

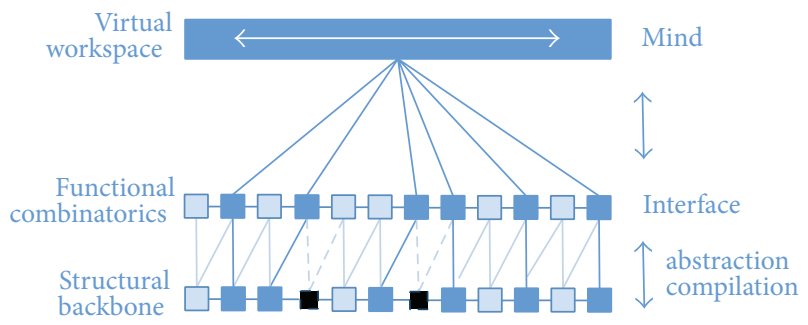

FIGURE 9: Structure-function relationships, abstraction layers and resilience. Beyond the notion of redundancy in distributed brain systems, hypothesis of the existence of abstraction layers, that is, a neural code, contributing to resilience, involving a partial virtualization of the functional architecture underlying cognition (see text).

even perhaps present features as universal as the genetic code but for biological cognition. It remains an open question.

Standard anatomofunctional explanations - in which a strong and rigid relationship between structure and function is generally expected-will miss essential aspects of the neural correlate of the mind. Understanding the nature of such "computation" and how it relates to the brain will present many theoretical and methodological challenges to cognitive neuroscience. If true, to some extent, such a framework will automatically raise potential concerns regarding the validity, in this context, of neuroanatomical hypotheses involving specific critical regions, either localized or distributed over several locations. Indeed in such a framework, the link between the brain substrate and the most integrative aspects of the mind might have to be reconceived on the model of the flexible relationships existing between hardware and software in computer science. As just mentioned, this would reopen profound questions about neural coding and suggest an approach to neuroscience returning to models inspired from system engineering and computer design, which have become often considered as inappropriate to understand 
biological systems such as the brain as a whole $[16,145]$. No matter what the specifications of such computational relationships would turn out to be, it would seem like an impossible task to infer the putative "coding scheme" from empirical observations, looking at correlations between structure and function, even with a perfect spatial and temporal resolution. In this respect it would be analogous to the impossible task of inferring the "operating system" running through a CPU by measuring the temperature of the CPU at multiple locations (which is not that much different from what we do when we use functional imaging or macroscopic electrophysiology in cognitive neuroscience). Nevertheless this is a task that neuroscience might eventually have to take on again, perhaps with the help of new theoretical breakthrough and computational resources. Models that are driven by first principles (such as Friston's free-energy driven Bayesian scheme for the brain; see $[139,140]$ ) might help to resolve this issue, and there is a strong need for progress in theoretical neuroscience and psychology.

\subsection{Is the Mind a Virtual Machine Running on a Distributed} Computer? We have envisioned in the previous section a series of arguments in support of the hypothesis that the mind relates to the brain through layers of abstraction, similar to what is implemented in computer science. We argued for the existence of low-level interfaces that govern the relationships between the rigid structural organization of the brain and information processing in neural networks, based on abstract combinatorial rules. The existence of such interfaces is suggested by the process of renormalization of information processing patterns that is observed in cognitive resilience to neurological insult, and unfolds while structural connectivity remains invariant. Such process and its top-down driving demonstrate the existence of degrees of freedom and of a relative independence between structure and function in the brain.

Similar features are found in virtual machines, or more generally in virtualization of distributed computer resources [146, 147]. "Virtualization" is a strategy aimed at simplifying and rendering more resilient interactions between systems, applications, or end users and complex distributed computing resources and devices, operating with rigidly set rules. It involves a set of operations that are precisely and explicitly aimed at hiding the physical characteristics or complicated details of an underlying hardware implementation, and at mapping function over structure flexibly, so that higher-order processes to which they are transparent can continue to operate independently of changes in the underlying hardware. The functional environment that is thereby created facilitates a resilient interfacing with other resources, by reducing dependency with the lower-layers of implementation down to the microdesign of the hardware. It allows the system to transfer entire processes from one physical environment to the other. It is used to facilitate multiplexed access, to combine resources at different physical locations under one virtual interface, and more generally to simplify the control of a complex device (in our case, the brain and the body in interaction with their environment). It generally takes many more cycles of computation to simulate resources and run a virtual machine than it would with a direct hardware implementation, so a virtualized environment can be slower, for equivalent amounts of nominal resources. For sure, controlled cognition and more generally higher-order cognition are slow (e.g., in the second range), as compared to electrochemical signaling (e.g., in the millisecond range). Virtualization is capable of providing maximum resilience to processes operating over networks of distributed computing resources. A form of virtualization of computation in the brain would thus appear to be a sound solution to resilience from an evolutionary standpoint.

Such a hypothesis entails conceptualizing the "mind" itself as a virtual entity (Figure 9) and the brain as a code interpreter. Without entering in the details of other reports in preparation, there is undeniable evidence that the brain is a code and command interpreter. The mere existence and the properties of language are proofs that this is the case at some level. Language, with its ability to address semantic memory and evoke mental imagery, is both one of the core components of higher-order cognition and a sparse, sequential code with specific combinatory rules. It is governed by universal syntactic principles and multiple layers of abstraction and parsing rules that are hierarchically nested (e.g., phonemes, morphemes, words, phrases, propositions, and so on). It can be dematerialized and carried via multiple media (e.g., speech and writing). In parsing, interpreting and understanding words, we are the living proof and we manifest that the brain is a code interpreter.

3.9. Resilience: Between Abstraction and Resistance. In this framework, irrespective of what the principles and details of the mind-brain interfacing may ultimately turn out to be, the mind is conceived as a functional entity. Through the phenomenon of cognitive resilience, this functional entity therefore behaves as an assaulted "fortress" which attempts to defend itself against desiccation and loss of autonomy. It struggles to rebuild its functional environment, even as its shrinking neural environment forces it to take adaptive measures. This is implicit and pervasive throughout the struggle and utmost distress of patients affected by AD as the night of dementia takes over and consciousness shrinks on a background of apathy and confusion. More generally, over its lifetime, the mind struggles with and resists insults and multiple normal and/or pathological perturbations arising from its neural, psychological, and social environments. The experience of effortful cognition, emotion regulation, willpower, and resilience to life events and disease is direct evidence of this existential fact.

Searching in brain mechanisms for the expression of this self-same resistance to destruction which is embedded at the core of cognition can be a strategy for isolating key aspects of the neural correlates of the mind. Given the core role of higher-order cognition itself, for example, self-awareness, in enabling and driving cognitive resilience and functional renormalization (see above), and more generally adaptive behaviors, it is intriguing to realize that it is possible to conceive of the very function of consciousness as being, in and of itself, a form of fundamental algorithm for resilience selected by evolution. 
At the core of this resilience, there is also a dimension of "resistance." The concept of resistance here is to be interpreted as a bridge-concept between phenomenology and physics. Brain computation and resilience encounter inertia in biological systems [16], including the inertia of synaptic plasticity, which slow down functional processes and render them as energetically costly. My hypothesis is that this plays a central role in shaping the phenomenology of the mind. This inner resistance of the mind against inertia that resilience manifests suggests that the mind also features a behavior of perturbed dynamical system: it drains energy to maintain its dynamics in a timely manner within certain boundaries of its state space based on an adaptive tuning of its control parameters (see $[12,16,17,136])$. As far as the mind is concerned, abstract computation thus seems to be intimately coupled with the physics of out-of-equilibrium systems.

For the longest, schools in the cognitive science opposed two views. On the one hand, there was the computational view on mind-brain relationships, sometimes construed as "ancient" with its software-hardware analogy and its emphasis on symbolic processing; what we could call the Von Neumann's brain. On the other hand, there was a more "modern" biophysical view on mindbrain relationships, which brings forth self-organization, complexity, and dynamical system theories, and relies implicitly or explicitly on the generic model of coupled differential equations and the notion of emergence, what we could call the Varela's brain (see $[136,148$, 149]). This academic antinomy was not in the mind of von Neumann when he developed his famous architecture at the core of all modern computers [150], as he based his reflections upon the architecture of the brain and the abstract model of neuron by McCulloch and Pitts [151], and derived the principles of implementation of universal arithmetical operations based on simple accumulators functioning as logical gates, by reasoning about small networks of interconnected formal neurons.

A network of computers-such as the Internet-is in turn certainly a complex, distributed dynamical system, embedding much self-organization (we need only consider all the various "bots" and autonomous agents trawling the web today), myriads of coupled oscillators at numerous scales, all while being run ultimately through the combinatory principles of a Von Neumann architecture. The possibility that the brain embeds similar principles should (re-)open many directions of research and therefore should be an opportunity to bring together several scientific communities that may have lost the habit of working together, or at the very least, that of cross-pollinating their research findings in ways that would ultimately prove mutually beneficial.

In summary, according to such a view, our mind is mediated by an algorithm that mimics a virtual machine and that governs the execution of an adaptive program in the brain, following inchoate and abstract combinatorial rules. The algorithm is characterized by principles that make the execution of such program resilient to structural damage, and more generally capable of coping, within the boundaries of energetic and chronometric constraints, with the inertia of its own biophysical implementation to produce adaptive cognition and behaviors. Behind a resilient mind there is a computation depending on a capacity to control routing and information transfer among distributed resources by building new paths of least resistance in the brain substrate. This requires both abstract principles and energy.

It is intriguing to consider that if evolution implies selecting resilient systems, in such framework, the ability to relate function and structure via layers of abstraction, or more generally abstraction, might paradoxically appear as a summit of biological evolution. It is further intriguing to consider that if functionally, consciousness itself is a form of higherorder, universal algorithm for resiliency, consciousness might itself appear to be the pinnacle of biological evolution qua abstraction. It is relevant to reemphasize here that basic consciousness appears to be one of the most resilient feature of cognition (see first part of the paper), as would be predicted based on the rationale that a mechanism optimized for the resiliency of cognitive and control systems should itself be maximally resilient (see also [12]).

\section{Studying the Mechanisms of Resilience: A Challenge for Neuroscience and Neuroimaging Methods}

One important venue for research on cognitive resilience is the study of structure-function relationships and their degrees of freedom at the large scale level. Several methodological challenges can be distinguished regarding this endeavor.

The Challenge of Multimodality. The disease processes and neurological disorders that manifest and call for cognitive resilience in patients struggling with their condition are diverse and complex. Their physiopathology often affects the brain at multiple scales and in multiple ways. The relative disconnect, extensively discussed above, between clinical expression and gross structural neuropathology that exists in resilient patients suggests the importance of specifically studying (1) functional parameters related to large-scale interactions and metabolism, in reference to (2) structural parameters, related to structural connectivity and morphometry (e.g., neuronal count). In order to (1) increase specificity, (2) adequately map this uncharted territory of compensatory anatomofunctional reorganizations, and (3) test hypotheses in a well-controlled, meaningful and conclusive way, we should endeavor to take into account the various levels of structural and metabolic brain alterations affecting the patients.

It is particularly important to find powerful and reliable solutions to analyze multimodal neuroimaging data together, as well as multiple dependent parameters. Thereby we can open multiple windows on one underlying brain. Contemporary medical imaging and multicentric projects foster the simultaneous use of complementary imaging modalities over a large number of patients at multiple time points. However solutions for proper data integration still need to be discovered and implemented in order to reduce the risk of data cemeteries, with an enormous amount of data that might not be exploited to their full potential. 
Multimodality can increase sensitivity and specificity, and it can offer better inferential power and better potential for pathophysiological interpretation [57]. It is particularly relevant for studying large-scale, higher-order, neurodynamical phenomena with specificity [67]. For instance, matching brains based on gray matter atrophy and accounting for structural disconnection and baseline metabolism are essential if one is to study which aspects of residual functional connectivity could account for CR specifically (see [8]). For example, significant differences in residual functional connectivity in fMRI between AD patients and healthy controls remained after accounting for structural damage such as cortical atrophy [152]. This suggests that it is important to extract information that is specific to each modality by taking into account the shared variance with the others. Yet studies combining structural and functional connectivity analyses are still in their infancy [66].

Multimodality is also important for understanding the relationships between different levels of observation in the brain, as well as making causal inferences about them. For instance, hippocampal atrophy in $\mathrm{AD}$ has been shown to be related to cingulum bundle atrophy, itself correlated with hypometabolism of the posterior cingulate cortex [153]. This suggested that the observed hypometabolism was related to the impact of hippocampal atrophy and mediated by the cingulum.

The Challenge of Integrating Longitudinal Information and Dynamics. Beyond multimodality, it is important to integrate a longitudinal perspective. Resilience is by definition a dynamical process that unfolds over time and at time scales that can vary greatly among disease processes and individuals. It can manifest through very rapid compensations over a matter of hours or days following certain strokes (e.g., the resolution of several acute-phase deficits like certain forms of anosognosia and aphasia) as well as through decades of struggle in the case of neurodegenerative processes such as $\mathrm{AD}$.

Thus within its course, AD presents fluctuations that could contain critical information about nonlinear transitions between stages of disease progression [154], reflecting critical neuropathological processes, mechanisms of vulnerability, and resilience. Therefore, it is important to study such processes using longitudinal paradigms, which can also yield an increase in sensitivity, specificity, and physiopathological interpretability, as well as enhance the translational impact of research on individual patients.

Integration of longitudinal data in discriminant analyses has proven to ameliorate early estimates of conversion from $\mathrm{MCI}$ to $\mathrm{AD}$ up to 6 months before conversion [155]. Given the high interindividual variability in clinical response to neuropathological processes, being able to model and account for within-subject variability can substantially improve the heuristic value of studies and signal detection. This view of the importance of longitudinal analyses is largely shared by the research community $[8,78]$. Many research groups from around the world are implementing large-scale multimodal longitudinal studies, for example, the Alzheimer's disease neuroimaging initiative (ADNI) [57], which includes more than 800 healthy elderly participants, MCI and AD patients. A further important one is the French MEMENTO project. Nevertheless few longitudinal studies in functional connectivity have been conducted in $\mathrm{AD}[66,67]$. Some longitudinal studies suggest an accelerated aging, a central role of the DMN and a driving role of the posterior cingulate cortex connectivity pattern in disease progression (see $[94,156])$.

Of course, the ideal rate of longitudinal acquisition should be adjusted to the "natural" rate of evolution that is specific to the neurological disorder or process under consideration. For instance, in awakening from coma, the ability to acquire data several times a week over the relatively short period during which there is a maximum expectation of change in mental status in the patients, would bring new fundamental insights about the mechanisms of recovery from the disorder, and have an impactful and lasting translational value. Of course, there are major practical issues of feasibility in such projects, due to safety concerns and clinical constraints, such as problems with titration as well as a lack of adequate infrastructures.

Several other important challenges face research about the dynamics of structure-function relationships. It is paramount to have well-controlled, basic research on the impact of experimental lesions of specific systems of fiber tracts and cortical regions on structural and functional connectivity over time. We must also look at the potential of electrical stimulation and pharmacology to renormalize functional connectivity, cognition and behavior following lesions. For these reasons, it is very important to support the development of valid, imageable, well-documented animal models, (e.g., macaque fascicularis and rat). A multitude of questions remains unanswered that only animal experiments on the topic will be able to address. A solid ground of basic research is necessary for interpreting the mass of multicentric, exploratory, observational human neuroimaging studies that are acquired everywhere in the world at great cost. Likewise, it is important to develop a sound computational framework in order to handle the different levels of observations involved, and quantify and model large-scale structurefunction relationships (Figures 2, 4, and 10). New multimodal multidimenional metrics will have to be developed, based on well-conceived models, in order to provide new biomarkers of disease processes and compensatory mechanisms with maximum sensitivity and specificity. Last but not least, there is the challenge of defining and implementing a heuristic framework to develop new strategies of treatments and solutions of renormalization of function. Such framework should be based on sound physiopathological and resilience-related mechanisms, derived from observation and modeling, and aimed at cognitive/behavioral rehabilitation strategies, electromagnetic stimulations, surgery and/or pharmacology.

\section{Perspectives for Medicine}

The relative disconnect between the clinical and neuropathological aspects of various neurological disorders, both within individuals across time and between individuals, has puzzled the research and clinical communities. Resilience is a central, positive contributor to such disconnect. By remaining 


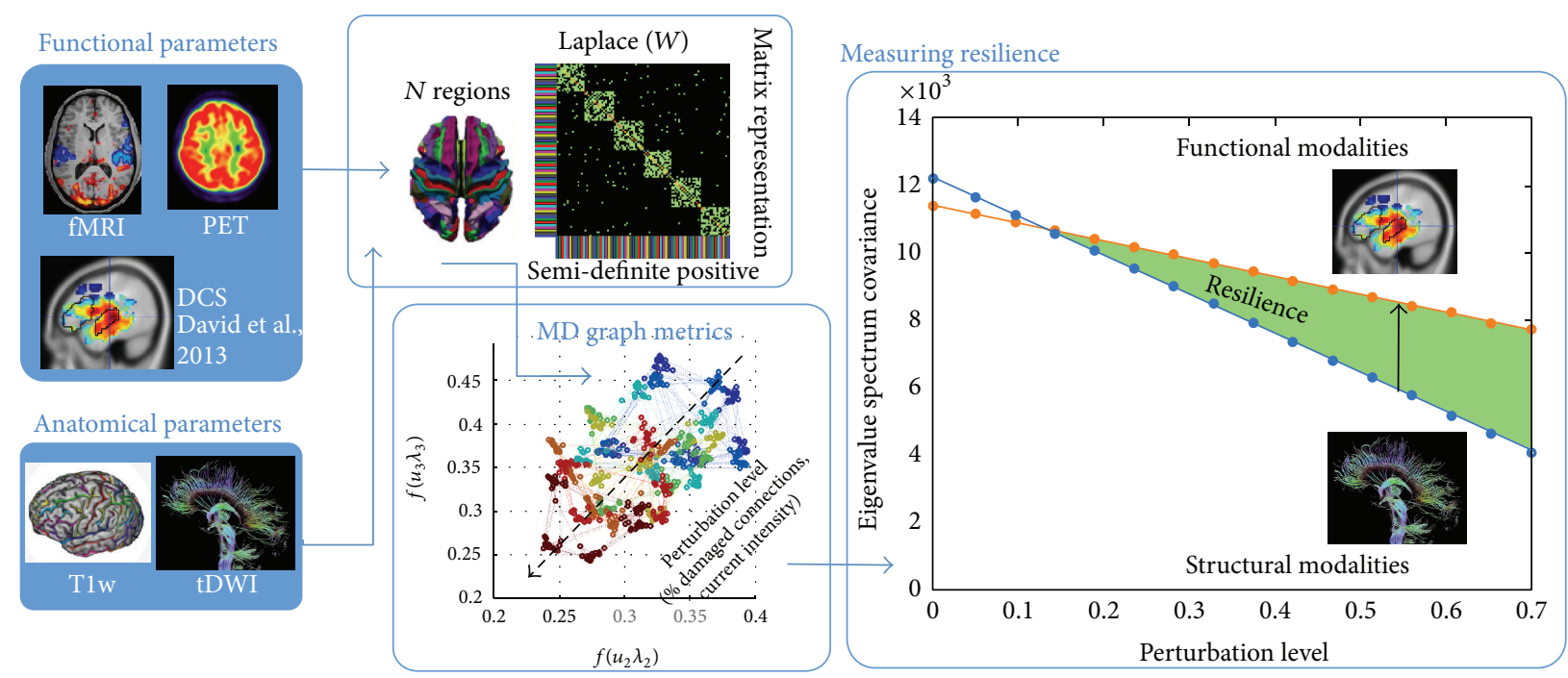

FIGURE 10: Addressing the challenges of quantifying resilience. Schema of a multimodal framework that distinguishes functional and anatomical parameters for key neuroimaging and electrical modalities. One approach is to compute matrices of connectivity based on neuroimaging signals, and then compute their Laplacian in order to obtain matrices that are semi-definite positive. Eigenvector decompositions of the matrices enable the analysis of their topological properties within multidimensional (MD) vector spaces with welldefined bases. Relations between modalities as well as the effect of structural perturbations can be measured in these spaces (see also Figure 4). Resilience can then be studied, modeled, and measured by computing parameters over these spaces and by comparing structural and functional modalities. In the framework discussed in this paper, one working hypothesis would be that resilient patients will demonstrate functional parameters that better resist damage (perturbation), in reference to normative atlases, than corresponding structural parameters.

unmodeled, it challenges the aim of achieving specificity and sensitivity in the estimation of risk of conversion to disease, differential diagnostic, prognostic, outcome of interventions, biomarker identification, the design of inclusion criteria for clinical trials aiming at reducing sample size, and that of prospective studies in which early detection of disease and testing disease-modifying treatments are central $[8,9]$.

The study and modeling of the mechanisms underlying resilience will thus contribute to increase specificity and sensitivity in the characterization of clinical and neuropathological relationships, by better modeling within-individual and interindividual variability. It will be a basis for defining new biomarkers, derived based on neurophysiologically sound hypotheses and well-behaved metrics over multimodal parameters, for better diagnostic, prognostic, and treatment outcome monitoring. Likewise, a better understanding of resilience will help to improve predictions regarding the clinical evolution of cognition and behavior based on multimodal neuroimaging. It will also offer a paradigm for the search for treatments and solutions in functional rehabilitation, and more generally interventional neurology, based on an understanding of the mechanisms enabling and fostering resilience.

Moreover the mechanisms supporting resilience might in certain cases directly alter the disease processes themselves, and not just compensate for them by providing a sheer buffer against the negative impact of the pathology on cognition. This disease-modifying effect might in part be mediated by neuromodulatory factors with anti-inflammatory and antiamyloid burden effects [131]. Exercise and cognitively stimulating experiences, notably the experience of novelty, directly impact the brain by increasing local brain volume, neurogenesis, synaptogenesis, the release of the brain derived neurotrophic factor (BDNF), and resistance to apoptosis. This ensemble of neuroprotective mechanisms might be fostered by increasing the noradrenergic tone, which according to recent theories could play a central role in CR [131]. Likewise, environmental enrichment has been suggested in animal models to prevent or slow $\mathrm{AD}$ beta amyloid pathology, suggesting a disease-modifying effect of factors related to CR [157]. Moreover, a relationship between higher CR and lower incidence or severity of anosognosia has been shown in dementia [78], which in turn suggests that fostering CR and self-awareness might help to contribute to reduce the risk of developing $\mathrm{AD}$.

In such a framework, research on the mechanisms of resilience and on possible treatments and interventions should strategically place some emphasis on computational modeling, building upon the potential for structure-function dissociations in the brain, and exploiting multimodal imaging to estimate relevant parameters and compare results to empirical observations. The question then can become that of the molecular and/or electrical mechanisms that could be introduced in the system as modeled (e.g., with a particular set of pathological biomarkers), in order to renormalize functional integration and cognition. A Big Data approach can help to derive priors about key features of target resilient versus nonresilient systems, which can in turn inform analyses and inferences, taking advantage for instance of web APIs. For example, one can perform automated metaanalyses of fMRI activation studies, based on text-mining and automated activation peak coordinate extraction from 
thousands of relevant references, in order to inform the characterization of the functional role of target systems (see http://neurosynth.org/; http://www.linkrbrain.org/; see also $[14,158])$. One can also analyze data of genetic expression from microarrays sampled over the brain in order to identify molecular footprints characteristic of target systems (e.g., Allen Brain Atlas: http://human.brain-map.org). For instance, Goel et al. [159] have recently demonstrated the existence of clear correlations between structural connectivity and genome-wide genetic expression data. Such a modeldriven approach will contribute to inform pharmacological research for treatments as well as possible solutions of surgery and electrostimulations. Likewise, better understanding the connections between cognition and the brain in such a framework will help to conceive functional rehabilitation strategies promoting resilience, based on cognitive stimulations and principles of computational psychology.

Finally, and importantly, adequate understanding and modeling of the mechanisms of resilience will also constitute an important basis for a reflection on preventive medicine in the domain of public health. This will help to identify factors and mechanisms for reducing the risk of disease onset or progression and for mitigating the functional impact of damage. Approaching brain disorders from the angle of resilience is developing a positive approach to medicine in the face of what cannot yet be cured.

Figure 11 summarizes the different scientific problems concerning the study of cognitive resilience to neurological insults and their organic relationships.

\section{Conclusion}

The present review and discussion support the hypothesis that higher-order cognition can be a highly robust, resilient, and flexible phenomenon in the face of extensive brain damage. A global process of renormalization of effective connectivity following a major disruption of structural connectivity can account for the rerouting of brain signal and for the effective preservation of the scheme of information processing subtending cognition. Bottom-up and top-down mechanisms, as well as local and global principles, are at play in the process. Self-awareness itself and more generally consciousness appear to be central in enabling top-down processes that are key for recovering impaired cognitive functions. We hypothesized that consciousness itself can be interpreted as a general algorithm for resilience selected by evolution. Progress in cognitive neuroscience of higherorder cognition might require considering computational hypotheses about brain-mind relationships and structurefunction relationships in the brain, in which the standard anatomofunctional framework would need to be complemented by an understanding of the putative layers of abstraction and principles of virtualization that may be at work in neurocognitive computation. These layers would not directly and simply be reflected in the brain hardware. Their nature remains mysterious, but the place of abstraction in nature might be greater than one thought.

The study and modeling of the mechanisms of resilience are important in order to increase specificity in diagnostic,

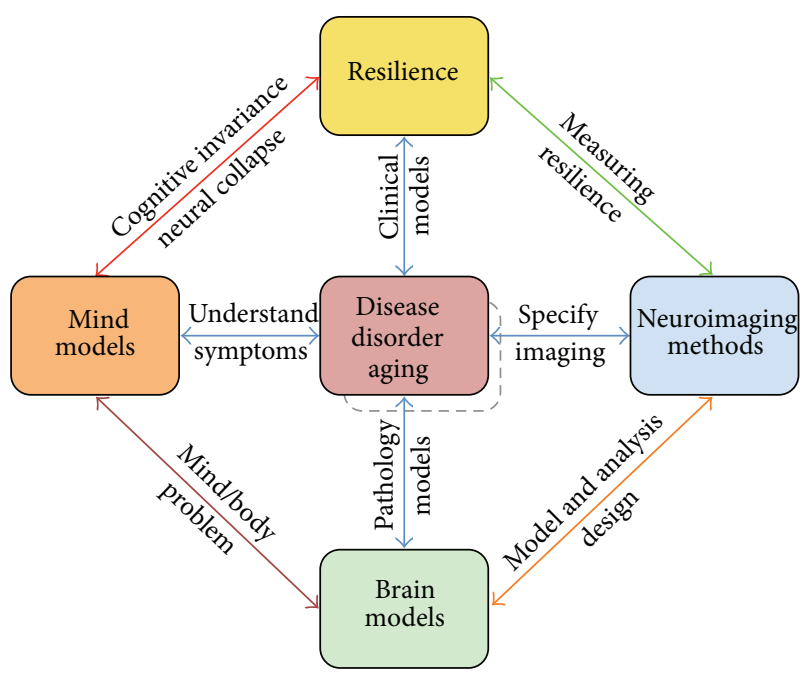

FIGURE 11: Relationships between levels of knowledge and scientific issues in the study and control of resilience. Roadmap for research on resilience. This diagram shows different scientific problems that are central to the study of cognitive resilience to neurological insults and their organic relationships.

prognostic, and treatment outcome monitoring in medicine. They also have a strong potential for informing prevention and treatment strategy, as well as technologies aimed at mitigating the functional impact of damage based on the renormalization of functions. More generally they could inform solutions to foster cognitive resilience against aging, as well as cognitive enhancement. They also open important perspectives for neuroengineering and artificial intelligence.

\section{Definitions}

Resilience. The ability for a system to resist dynamically a perturbation or adverse condition that challenges the integrity of its normal operation and to preserve function as a result in reference to some initial design or normative functional standards.

Structural Connectivity. Informs the physical connections (e.g., fiber tracts or axons) existing between pair of nodes (e.g., brain regions or neurons) in a network, at a given scale. For the brain, it is often estimated in vivo based on tractography over diffusion weighted imaging (DWI). The analysis of a matrix of structural connectivity can identify direct and indirect, alternate structural pathways between regions.

Effective Connectivity. An indicator of presence of structural connectivity weighted by a transmission or synaptic gain. This parameter regulates signal propagation and interactions between network nodes (neurons or brain regions, depending on the scale considered). It is equivalent to the concept of synaptic weight at the synaptic scale but can be used more generally to characterize the gain of transmission between 
network nodes at any scale. It is an essential level of modeling of functional plasticity in the brain.

Functional Connectivity. A measure of the correlations between brain signals, often empirically derived from resting state functional magnetic resonance imaging (rsfMRI) data. It reflects both direct and indirect interactions between network nodes (e.g., brain regions). It is formally similar to analyses of coherence performed on electrophysiological signals. Functional connectivity relates to structural and effective connectivity through a generative process or model of brain dynamics based on physiological mechanisms.

Mutual Information. A quantity derived from Shannon's entropy, which characterizes the degree of statistical dependence between two variables $X$ and $Y$. It is minimal when two systems do not exchange information and maximum when they share the exact same information. It reflects both direct and indirect information transfer (e.g., via indirect pathways) between network nodes (e.g., brain regions). Mutual information relates to structural and effective connectivity through a generative process or model of brain dynamics based on physiological mechanisms.

Degeneracy. Form of redundancy in networks. Multiple alternate available processing units/nodes are capable of handling the same function (e.g., parallel array of identical specialized processors).

Pluripotency. Form of redundancy in networks. Processing units/nodes are individually capable of handling multiple functions contextually (e.g., processor individually executing different tasks sequentially by switching between them).

Disconnection. A disconnection of pathways in the matrix of structural connectivity following brain damage. Disconnection disrupts communication and interactions between network nodes (e.g., brain regions) normally connected and interacting via these pathways.

Routing. The mechanism regulating the transfer of information between network nodes along existing structural connections to preserve the timely execution of functional processes that critically depend on the interaction between these nodes. Dynamic routing in the brain can be controlled by modulating effective connectivity between network nodes (e.g., brain regions) along structural pathways.

Renormalization of Function. Relative preservation of or return to the "normal," overall functional scheme underlying normal cognition, following brain damage, in a manner that is compatible with residual structural and metabolic resources. It is the problem that a resilient brain must resolve after brain damage.

\section{Conflict of Interests}

The author declares that there is no conflict of interests regarding the publication of this paper.

\section{Acknowledgments}

The author would like to particularly thank Ralph Adolphs, Justin Feinstein, Katharina Orhnberger, Guillaume Marrelec, Kenneth Williford, and ZZ Packer for their invaluable feedbacks during the completion of this paper. He also thanks Dirk Neumann, Alain Giron, and Mélanie Pélégrini for their insights. Additionally the author would like to thank for their direct or indirect influence on these ideas over the years: F. Varela, A. Damasio, Th. Grabowski, D. Tranel, S. Metha, S. Khalsa, C. Philippi, H. Benali, G. Landini, Y. Burnod, D. Bennequin, and M. Le Van Quyen.

\section{References}

[1] A. Luria, The Man with a Shattered World: The History of a Brain Wound, Harvard University Press, Cambridge, Mass, USA, 1987.

[2] U. Noppeney, K. J. Friston, and C. J. Price, "Degenerate neuronal systems sustaining cognitive functions," Journal of Anatomy, vol. 205, no. 6, pp. 433-442, 2004.

[3] C. L. Philippi, J. S. Feinstein, S. S. Khalsa et al., "Preserved self-awareness following extensive bilateral brain damage to the insula, anterior cingulate, and medial prefrontal cortices," PLoS ONE, vol. 7, no. 8, Article ID e38413, 2012.

[4] S. Laureys, M. Boly, and P. Maquet, "Tracking the recovery of consciousness from coma," Journal of Clinical Investigation, vol. 116, no. 7, pp. 1823-1825, 2006.

[5] A. M. Owen, M. R. Coleman, M. Boly, M. H. Davis, S. Laureys, and J. D. Pickard, "Detecting awareness in the vegetative state," Science, vol. 313, no. 5792, p. 1402, 2006.

[6] M. M. Monti, A. Vanhaudenhuyse, M. R. Coleman et al., "Willful modulation of brain activity in disorders of consciousness," The New England Journal of Medicine, vol. 362, no. 7, pp. 579589, 2010.

[7] O. Sacks, Awakenings, Pelican Books, Harmondsworth, UK, 1976.

[8] Y. Stern, “Cognitive reserve," Neuropsychologia, vol. 47, no. 10, pp. 2015-2028, 2009.

[9] B. Dubois, H. H. Feldman, C. Jacova et al., "Revising the definition of Alzheimer's disease: a new lexicon," The Lancet Neurology, vol. 9, no. 11, pp. 1118-1127, 2010.

[10] M. F. de Oliveira, F. C. G. Pinto, K. Nishikuni, R. V. Botelho, A. M. Lima, and J. M. Rotta, "Revisiting hydrocephalus as a model to study brain resilience," Frontiers in Human Neuroscience, vol. 5, article 181, 2012.

[11] Y. Stern, "Cognitive reserve and Alzheimer disease," Alzheimer Disease and Associated Disorders, vol. 20, no. 2, pp. 112-117, 2006.

[12] K. Williford, D. Rudrauf, and C. L. Philippi, "Selfconsciousness," in Encyclopedia of the Mind, Sage, Thousand Oaks, Calif, USA, 2011.

[13] S. S. Khalsa, D. Rudrauf, J. S. Feinstein, and D. Tranel, "The pathways of interoceptive awareness," Nature Neuroscience, vol. 12, no. 12, pp. 1494-1496, 2009.

[14] G. M. Edelman and J. A. Gally, "Degeneracy and complexity in biological systems," Proceedings of the National Academy of Sciences of the United States of America, vol. 98, no. 24, pp. 13763-13768, 2001.

[15] R. G. Lee and P. van Donkelaar, "Mechanisms underlying functional recovery following stroke," Canadian Journal of Neurological Sciences, vol. 22, no. 4, pp. 257-263, 1995. 
[16] D. Rudrauf and A. Damasio, "A conjecture regarding the biological mechanism of subjectivity and feeling," Journal of Consciousness Studies, vol. 12, no. 8-10, pp. 236-262, 2005.

[17] S. Paradiso and D. Rudrauf, "Struggle for life, struggle for love and recognition: the neglected self in social cognitive neuroscience," Dialogues in Clinical Neuroscience, vol. 14, no. 1, pp. 65-75, 2012.

[18] W. Penfield, "Neurophysiological basis of the higher functions of the nervous system," in Handbook of Physiology; A Critical, Comprehensive Presentation of Physiological Knowledge and Concepts, L. S. Jefferson, A. D. Cherrington, and H. M. Goodman, Eds., vol. 6, p. 1254, Oxford University Press, New York, NY, USA, 1960.

[19] A. Damasio, The Feeling of What Happens: Body and Emotion in the Making of Consciousness, Harvest Books, 1999.

[20] L. Feuillet, H. Dufour, and J. Pelletier, "Brain of a white-collar worker," The Lancet, vol. 370, no. 9583, p. 262, 2007.

[21] H. Damasio and A. Damasio, Lesion Analysis in Neuropsychology, Oxford University Press, New York, NY, USA, 1989.

[22] D. Rudrauf, S. Mehta, J. Bruss, D. Tranel, H. Damasio, and T. J. Grabowski, "Thresholding lesion overlap difference maps: application to category-related naming and recognition deficits," NeuroImage, vol. 41, no. 3, pp. 970-984, 2008.

[23] D. Rudrauf, S. Mehta, and T. J. Grabowski, "Disconnection's renaissance takes shape: formal incorporation in group-level lesion studies," Cortex, vol. 44, no. 8, pp. 1084-1096, 2008.

[24] M. C. Cirstea and M. F. Levin, "Compensatory strategies for reaching in stroke," Brain, vol. 123, part 5, pp. 940-953, 2000.

[25] A. R. Damasio, Descartes' Error: Emotion, Reason and the Human Brain, Grosset/Putnam, New York, NY, USA, 1994.

[26] M. Catani and D. H. Ffytche, "The rises and falls of disconnection syndromes," Brain, vol. 128, no. 10, pp. 2224-2239, 2005.

[27] A. D. Craig, "Human feelings: why are some more aware than others?" Trends in Cognitive Sciences, vol. 8, no. 6, pp. 239-241, 2004.

[28] A. D. Craig, "How do you feel-now? The anterior insula and human awareness," Nature Reviews Neuroscience, vol. 10, no. 1 , pp. 59-70, 2009.

[29] R. Z. Goldstein, A. D. Craig, A. Bechara et al., "The neurocircuitry of impaired insight in drug addiction," Trends in Cognitive Sciences, vol. 13, no. 9, pp. 372-380, 2009.

[30] T. Singer, H. D. Critchley, and K. Preuschoff, "A common role of insula in feelings, empathy and uncertainty," Trends in Cognitive Sciences, vol. 13, no. 8, pp. 334-340, 2009.

[31] D. A. Gusnard, E. Akbudak, G. L. Shulman, and M. E. Raichle, "Medial prefrontal cortex and self-referential mental activity: relation to a default mode of brain function," Proceedings of the National Academy of Sciences of the United States of America, vol. 98, no. 7, pp. 4259-4264, 2001.

[32] A. W. M. Kelley, C. N. Macrae, C. L. Wyland, S. Caglar, S. Inati, and T. F. Heatherton, "Finding the self? An event-related fMRI study," Journal of Cognitive Neuroscience, vol. 14, no. 5, pp. 785794, 2002.

[33] D. M. Amodio and C. D. Frith, "Meeting of minds: the medial frontal cortex and social cognition," Nature Reviews Neuroscience, vol. 7, no. 4, pp. 268-277, 2006.

[34] C. L. Philippi, M. C. Duff, N. L. Denburg, D. Tranel, and D. Rudrauf, "Medial PFC damage abolishes the self-reference effect," Journal of Cognitive Neuroscience, vol. 24, no. 2, pp. 475481, 2012.
[35] J. S. Feinstein, D. Rudrauf, S. S. Khalsa et al., "Bilateral limbic system destruction in man," Journal of Clinical and Experimental Neuropsychology, vol. 32, no. 1, pp. 88-106, 2010.

[36] R. L. Buckner, J. R. Andrews-Hanna, and D. L. Schacter, "The brain's default network: anatomy, function, and relevance to disease," Annals of the New York Academy of Sciences, vol. 1124, pp. 1-38, 2008.

[37] A. Damasio, Self Comes to Mind, Constructing the Conscious Brain, Random House, 2011.

[38] A. Damasio, H. Damasio, and D. Tranel, "Persistence of feelings and sentience after bilateral damage of the insula," Cerebral Cortex, vol. 23, no. 4, pp. 833-846, 2013.

[39] J. S. Feinstein, "Lesion studies of human emotion and feeling," Current Opinion in Neurobiology, vol. 23, no. 3, pp. 304-309, 2013.

[40] J. S. Feinstein, C. Buzza, R. Hurlemann et al., "Fear and panic in humans with bilateral amygdala damage," Nature Neuroscience, vol. 16, no. 3, pp. 270-272, 2013.

[41] B. A. Wilson, M. Kopelman, and N. Kapur, "Prominent and persistent loss of past awareness in amnesia: delusion, impaired consciousness or coping strategy?" Neuropsychological Rehabilitation, vol. 18, no. 5-6, pp. 527-540, 2008.

[42] S. Laureys, J. T. Giacino, N. D. Schiff, M. Schabus, and A. M. Owen, "How should functional imaging of patients with disorders of consciousness contribute to their clinical rehabilitation needs?" Current Opinion in Neurology, vol. 19, no. 6, pp. 520527, 2006.

[43] S. Laureys and G. Tononi, The Neurology of Consciousness, Academic Press, London, UK, 2009.

[44] A. Owen, N. Schiff, and S. Laureys, "The assessment of conscious awareness in the vegetative state," in The Neurology of Consciousness, S. Laureys and G. Tononi, Eds., pp. 163-172, Academic Press, London, UK, 2009.

[45] M. Boly, L. Tshibanda, A. Vanhaudenhuyse et al., "Functional connectivity in the default network during resting state is preserved in a vegetative but not in a brain dead patient," Human Brain Mapping, vol. 30, no. 8, pp. 2393-2400, 2009.

[46] A. Vanhaudenhuyse, Q. Noirhomme, L. J.-F. Tshibanda et al., "Default network connectivity reflects the level of consciousness in non-communicative brain-damaged patients," Brain, vol.133, no. 1, pp. 161-171, 2010.

[47] J. Giacino and N. Schiff, "The minimally conscious state: clinical features, pathophysiology and therapeutic implications," in The Neurology of Consciousness, S. Laureys and G. Tononi, Eds., pp. 173-190, Academic Press, London, UK, 2009.

[48] J. T. Giacino and K. Kalmar, “The vegetative and minimally conscious states: a comparison of clinical features and functional outcome," Journal of Head Trauma Rehabilitation, vol. 12, no. 4, pp. 36-51, 1997.

[49] B. T. Hyman, H. Damasio, A. R. Damasio, and G. W. van Hoesen, "Alzheimer's disease," Annual Review of Public Health, vol. 10, pp. 115-140, 1989.

[50] C. R. Jack Jr., D. S. Knopman, W. J. Jagust et al., "Hypothetical model of dynamic biomarkers of the Alzheimer's pathological cascade," The Lancet Neurology, vol. 9, no. 1, pp. 119-128, 2010.

[51] J. Babinski, "Anosognosia," The Journal of Nervous and Mental Disease, vol. 51, article 70, 1920.

[52] S. Banks and S. Weintraub, "Self-awareness and self-monitoring of cognitive and behavioral deficits in behavioral variant frontotemporal dementia, primary progressive aphasia and probable Alzheimer's disease," Brain and Cognition, vol. 67, no. 1, pp. 58-68, 2008. 
[53] S. E. Starkstein, R. Jorge, R. Mizrahi, J. Adrian, and R. G. Robinson, "Insight and danger in Alzheimer's disease," European Journal of Neurology, vol. 14, no. 4, pp. 455-460, 2007.

[54] S. E. Starkstein, S. Brockman, D. Bruce, and G. Petracca, "Anosognosia is a significant predictor of apathy in Alzheimer's disease," Journal of Neuropsychiatry and Clinical Neurosciences, vol. 22, no. 4, pp. 378-383, 2010.

[55] G. Spalletta, P. Girardi, C. Caltagirone, and M. D. Orfei, "Anosognosia and neuropsychiatric symptoms and disorders in mild alzheimer disease and mild cognitive impairment," Journal of Alzheimer's Disease, vol. 29, no. 4, pp. 761-772, 2012.

[56] M. J. Al-Aloucy, R. Cotteret, P. Thomas, M. Volteau, I. Benmaou, and G. Dalla Barba, "Unawareness of memory impairment and behavioral abnormalities in patients with Alzheimer's disease: relation to professional health care burden," Journal of Nutrition, Health and Aging, vol. 15, no. 5, pp. 356-360, 2011.

[57] M. W. Weiner, D. P. Veitch, P. S. Aisen et al., "The Alzheimer's disease neuroimaging initiative: a review of papers published since its inception," Alzheimer's and Dementia, vol. 8, supplement 1, pp. S1-S68, 2012.

[58] S. E. Arnold, B. T. Hyman, J. Flory, A. R. Damasio, and G. W. van Hoesen, "The topographical and neuroanatomical distribution of neurofibrillary tangles and neuritic plaques in the cerebral cortex of patients with alzheimer's disease," Cerebral Cortex, vol. 1, no. 1, pp. 103-116, 1991.

[59] M.-C. de Lacoste and C. L. White III, "The role of cortical connectivity in Alzheimer's disease pathogenesis: a review and model system," Neurobiology of Aging, vol. 14, no. 1, pp. 1-16, 1993.

[60] S. M. Landau, D. Harvey, C. M. Madison et al., "Associations between cognitive, functional, and FDG-PET measures of decline in AD and MCI," Neurobiology of Aging, vol. 32, no. 7, pp. 1207-1218, 2011.

[61] J. Parvizi, G. W. van Hoesen, and A. Damasio, "Severe pathological changes of parabrachial nucleus in Alzheimer's disease," NeuroReport, vol. 9, no. 18, pp. 4151-154, 1998.

[62] J. Parvizi, G. W. van Hoesen, and A. Damasio, "Selective pathological changes of the periaqueductal gray matter in Alzheimer's disease," Annals of Neurology, vol. 48, no. 3, pp. 344-353, 2000.

[63] X. Delbeuck, M. van der Linden, and F. Collette, "Alzheimer's disease as a disconnection syndrome?" Neuropsychology Review, vol. 13, no. 2, pp. 79-92, 2003.

[64] X. Delbeuck, F. Collette, and M. van der Linden, "Is Alzheimer's disease a disconnection syndrome?. Evidence from a crossmodal audio-visual illusory experiment," Neuropsychologia, vol. 45, no. 14, pp. 3315-3323, 2007.

[65] Y. He, Z. Chen, G. Gong, and A. Evans, "Neuronal networks in Alzheimer's disease," Neuroscientist, vol. 15, no. 4, pp. 333-350, 2009.

[66] M. Filippi and F. Agosta, "Structural and functional network connectivity breakdown in Alzheimer's disease studied with magnetic resonance imaging techniques," Journal of Alzheimer's Disease, vol. 24, no. 3, pp. 455-474, 2011.

[67] T. Xie and Y. He, "Mapping the Alzheimer's brain with connectomics," Frontiers in Psychiatry, vol. 2, article 77, 2012.

[68] S. Neufang, A. Akhrif, V. Riedl et al., "Disconnection of frontal and parietal areas contributes to impaired attention in very early Alzheimer's disease," Journal of Alzheimer's, vol. 25, no. 2, pp. 309-321, 2011.
[69] M. Pievani, F. Agosta, E. Pagani et al., "Assessment of white matter tract damage in mild cognitive impairment and Alzheimer's disease," Human Brain Mapping, vol. 31, no. 12, pp. 1862-1875, 2010.

[70] J. L. Price and J. C. Morris, "Tangles and plaques in nondemented aging and "preclinical" Alzheimer's disease," Annals of Neurology, vol. 45, no. 3, pp. 358-368, 1999.

[71] Neuropathology Group of the Medical Research Council Cognitive Function and Ageing Study (MRC CFAS), "Pathological correlates of late-onset dementia in a multicentre, communitybased population in England and Wales," The Lancet, vol. 357, no. 9251, pp. 169-175, 2001.

[72] J. Steffener and Y. Stern, "Exploring the neural basis of cognitive reserve in aging," Biochimica et Biophysica Acta: Molecular Basis of Disease, vol. 1822, no. 3, pp. 467-473, 2012.

[73] Y. Stern, B. Gurland, T. K. Tatemichi, M. X. Tang, D. Wilder, and R. Mayeux, "Influence of education and occupation on the incidence of Alzheimer's disease," Journal of the American Medical Association, vol. 271, no. 13, pp. 1004-1010, 1994.

[74] M. J. Valenzuela and P. Sachdev, "Brain reserve and dementia: a systematic review," Psychological Medicine, vol. 36, no. 4, pp. 441-454, 2006.

[75] H. Braak and E. Braak, "Neuropathological stageing of Alzheimer-related changes," Acta Neuropathologica, vol. 82, no. 4, pp. 239-259, 1991.

[76] C. M. Roe, M. A. Mintun, G. D’Angelo, C. Xiong, E. A. Grant, and J. C. Morris, "Alzheimer disease and cognitive reserve: variation of education effect with carbon 11-labeled pittsburgh compound B uptake," Archives of Neurology, vol. 65, no. 11, pp. $1467-1471,2008$.

[77] D. A. Bennett, R. S. Wilson, J. A. Schneider et al., "Education modifies the relation of AD pathology to level of cognitive function in older persons," Neurology, vol. 60, no. 12, pp. 19091915, 2003.

[78] M. B. Spitznagel and G. Tremont, "Cognitive reserve and anosognosia in questionable and mild dementia," Archives of Clinical Neuropsychology, vol. 20, no. 4, pp. 505-515, 2005.

[79] J. Gläscher, D. Rudrauf, R. Colom et al., "Distributed neural system for general intelligence revealed by lesion mapping," Proceedings of the National Academy of Sciences of the United States of America, vol. 107, no. 10, pp. 4705-4709, 2010.

[80] R. Chen, L. G. Cohen, and M. Hallett, "Nervous system reorganization following injury," Neuroscience, vol. 111, no. 4, pp. 761-773, 2002.

[81] G. Marrelec, A. Krainik, H. Duffau et al., "Partial correlation for functional brain interactivity investigation in functional MRI," NeuroImage, vol. 32, no. 1, pp. 228-237, 2006.

[82] A. R. Carter, S. V. Astafiev, C. E. Lang et al., "Resting interhemispheric functional magnetic resonance imaging connectivity predicts performance after stroke," Annals of Neurology, vol. 67, no. 3, pp. 365-375, 2010.

[83] E. M. Nomura, C. Gratton, R. M. Visser, A. Kayser, F. Perez, and M. D'Esposito, "Double dissociation of two cognitive control networks in patients with focal brain lesions," Proceedings of the National Academy of Sciences of the United States of America, vol. 107, no. 26, pp. 12017-12022, 2010.

[84] M. Boly, V. Perlbarg, G. Marrelec et al., "Hierarchical clustering of brain activity during human nonrapid eye movement sleep," Proceedings of the National Academy of Sciences of the United States of America, vol. 109, no. 15, pp. 5856-5861, 2012. 
[85] M. D. Fox, A. Z. Snyder, J. L. Vincent, M. Corbetta, D. C. van Essen, and M. E. Raichle, "The human brain is intrinsically organized into dynamic, anticorrelated functional networks," Proceedings of the National Academy of Sciences of the United States of America, vol. 102, no. 27, pp. 9673-9678, 2005.

[86] M. Boly, C. Phillips, E. Balteau et al., "Consciousness and cerebral baseline activity fluctuations," Human Brain Mapping, vol. 29, no. 7, pp. 868-874, 2008.

[87] F. Cauda, B. M. Micon, K. Sacco et al., "Disrupted intrinsic functional connectivity in the vegetative state," Journal of Neurology, Neurosurgery and Psychiatry, vol. 80, no. 4, pp. 429431, 2009.

[88] W. W. Seeley, V. Menon, A. F. Schatzberg et al., "Dissociable intrinsic connectivity networks for salience processing and executive control," Journal of Neuroscience, vol. 27, no. 9, pp. 2349-2356, 2007.

[89] V. L. Morgan, A. Mishra, A. T. Newton, J. C. Gore, and Z. Ding, "Integrating functional and diffusion magnetic resonance imaging for analysis of structure-function relationship in the human language network," PLoS ONE, vol. 4, no. 8, Article ID e6660, 2009.

[90] G. Lohmann, S. Hoehl, J. Brauer et al., "Setting the frame: the human brain activates a basic low-frequency network for language processing," Cerebral Cortex, vol. 20, no. 6, pp. 12861292, 2010.

[91] D. Zhang and M. E. Raichle, "Disease and the brain's dark energy," Nature Reviews Neurology, vol. 6, no. 1, pp. 15-28, 2010.

[92] F. Agosta, M. Pievani, C. Geroldi, M. Copetti, G. B. Frisoni, and M. Filippi, "Resting state fMRI in Alzheimer's disease: beyond the default mode network," Neurobiology of Aging, vol. 33, no. 8, pp. 1564-1578, 2012.

[93] C. J. Stam, W. de Haan, A. Daffertshofer et al., "Graph theoretical analysis of magnetoencephalographic functional connectivity in Alzheimer's disease," Brain, vol. 132, no. 1, pp. 213-224, 2009.

[94] H.-Y. Zhang, S.-J. Wang, B. Liu et al., "Resting brain connectivity: changes during the progress of Alzheimer disease," Radiology, vol. 256, no. 2, pp. 598-606, 2010.

[95] C. Sorg, V. Riedl, M. Mühlau et al., "Selective changes of restingstate networks in individuals at risk for Alzheimer's disease," Proceedings of the National Academy of Sciences of the United States of America, vol. 104, no. 47, pp. 18760-18765, 2007.

[96] T. Nakamura, F. G. Hillary, and B. B. Biswal, "Resting network plasticity following brain injury," PLoS ONE, vol. 4, no. 12, Article ID e8220, 2009.

[97] D. J. Sharp, C. F. Beckmann, R. Greenwood et al., "Default mode network functional and structural connectivity after traumatic brain injury," Brain, vol. 134, no. 8, pp. 2233-2247, 2011.

[98] R. L. Buckner, J. Sepulcre, T. Talukdar et al., "Cortical hubs revealed by intrinsic functional connectivity: mapping, assessment of stability, and relation to Alzheimer's disease," Journal of Neuroscience, vol. 29, no. 6, pp. 1860-1873, 2009.

[99] J. S. Damoiseaux, C. F. Beckmann, E. J. S. Arigita et al., "Reduced resting-state brain activity in the "default network" in normal aging," Cerebral Cortex, vol. 18, no. 8, pp. 1856-1864, 2008.

[100] M. Ystad, E. Hodneland, S. Adolfsdottir et al., "Cortico-striatal connectivity and cognition in normal aging: a combined DTI and resting state fMRI study," NeuroImage, vol. 55, no. 1, pp. 2431, 2011.

[101] C. J. Honey, J.-P. Thivierge, and O. Sporns, "Can structure predict function in the human brain?" NeuroImage, vol. 52, no. 3, pp. 766-776, 2010.
[102] A. Messé, D. Rudrauf, H. Benali, and G. Marrelec, "Relating structure and function in the human brain: relative contributions of anatomy, stationary dynamics, and non-stationarities," PLoS Computational Biology, vol. 10, no. 3, Article ID e1003530, 2014.

[103] J. S. Damoiseaux and M. D. Greicius, "Greater than the sum of its parts: a review of studies combining structural connectivity and resting-state functional connectivity," Brain Structure \& Function, vol. 213, no. 6, pp. 525-533, 2009.

[104] L. Q. Uddin, E. Mooshagian, E. Zaidel et al., "Residual functional connectivity in the split-brain revealed with resting-state functional MRI," NeuroReport, vol. 19, no. 7, pp. 703-709, 2008.

[105] J. Michael Tyszka, D. P. Kennedy, R. Adolphs, and L. K. Paul, "Intact bilateral resting-state networks in the absence of the corpus callosum," Journal of Neuroscience, vol. 31, no. 42, pp. 15154-15162, 2011.

[106] M. J. Lowe, B. J. Mock, and J. A. Sorenson, "Functional connectivity in single and multislice echoplanar imaging using resting-state fluctuations," NeuroImage, vol. 7, no. 2, pp. 119-132, 1998.

[107] J. S. Damoiseaux, S. A. R. B. Rombouts, F. Barkhof et al., "Consistent resting-state networks across healthy subjects," Proceedings of the National Academy of Sciences of the United States of America, vol. 103, no. 37, pp. 13848-13853, 2006.

[108] Y. Nir, U. Hasson, I. Levy, Y. Yeshurun, and R. Malach, "Widespread functional connectivity and fMRI fluctuations in human visual cortex in the absence of visual stimulation," NeuroImage, vol. 30, no. 4, pp. 1313-1324, 2006.

[109] S. Chen, T. J. Ross, W. Zhan et al., "Group independent component analysis reveals consistent resting-state networks across multiple sessions," Brain Research, vol. 1239, pp. 141-151, 2008.

[110] B. J. He, A. Z. Snyder, J. L. Vincent, A. Epstein, G. L. Shulman, and M. Corbetta, "Breakdown of functional connectivity in frontoparietal networks underlies behavioral deficits in spatial neglect," Neuron, vol. 53, no. 6, pp. 905-918, 2007.

[111] A. del Cul, S. Dehaene, P. Reyes, E. Bravo, and A. Slachevsky, "Causal role of prefrontal cortex in the threshold for access to consciousness," Brain, vol. 132, no. 9, pp. 2531-2540, 2009.

[112] J. Wang, X. Zuo, and Y. He, "Graph-based network analysis of resting-state functional MRI," Frontiers in Systems Neuroscience, vol. 4, article 16, 2010.

[113] F. Bartolomei, I. Bosma, M. Klein et al., "Disturbed functional connectivity in brain tumour patients: evaluation by graph analysis of synchronization matrices," Clinical Neurophysiology, vol. 117, no. 9, pp. 2039-2049, 2006.

[114] F. Bartolomei, I. Bosma, M. Klein et al., "How do brain tumors alter functional connectivity? A magnetoencephalography study," Annals of Neurology, vol. 59, no. 1, pp. 128-138, 2006.

[115] S. C. Ponten, F. Bartolomei, and C. J. Stam, "Small-world networks and epilepsy: graph theoretical analysis of intracerebrally recorded mesial temporal lobe seizures," Clinical Neurophysiology, vol. 118, no. 4, pp. 918-927, 2007.

[116] S. D. Roosendaal, M. M. Schoonheim, H. E. Hulst et al., "Resting state networks change in clinically isolated syndrome," Brain, vol. 133, no. 6, pp. 1612-1621, 2010.

[117] D. Rudrauf, A. Douiri, C. Kovach et al., "Frequency flows and the time-frequency dynamics of multivariate phase synchronization in brain signals," NeuroImage, vol. 31, no. 1, pp. 209-227, 2006. 
[118] P. Comon and C. Jutten, Handbook of Blind Source Separation, Independent Component Analysis and Applications, Academic Press, Oxford, UK, 2010.

[119] D. O. Hebb, "Distinctive features of learning in the higher animal," in Brain Mechanisms and Learning, J. F. Delafresnaye, Ed., Oxford University Press, London, UK, 1961.

[120] K. I. Diamantras and S. Y. Kung, Principal Component Neural Networks, John Wiley \& Sons, New York, NY, USA, 1996.

[121] A. J. Bell and T. J. Sejnowski, "An information-maximization approach to blind separation and blind deconvolution," Neural Computation, vol. 7, no. 6, pp. 1129-1159, 1995.

[122] S. Mesmoudi, V. Perlbarg, D. Rudrauf et al., "Resting state networks' corticotopy: the dual intertwined rings architecture," PLoS ONE, vol. 8, no. 7, Article ID e67444, 2013.

[123] T. Cover and J. Thomas, Elements of Information Theory, John Wiley \& Sons, New York, NY, USA edition, 1991.

[124] C. E. Shannon, "A mathematical theory of communication," Bell System Technical Journal, vol. 27, no. 3, pp. 379-423, 1948.

[125] O. Sporns, "The human connectome: a complex network," Annals of the New York Academy of Sciences, vol. 1224, no. 1, pp. 109-125, 2011.

[126] N. T. Markov, M. Ercsey-Ravasz, D. C. van Essen, K. Knoblauch, Z. Toroczkai, and H. Kennedy, "Cortical high-density counterstream architectures," Science, vol. 342, no. 6158, Article ID 1238406, 2013.

[127] G. Tononi, "An information integration theory of consciousness," BMC Neuroscience, vol. 5, article 42, 2004.

[128] V. Mante, D. Sussillo, K. V. Shenoy, and W. T. Newsome, "Context-dependent computation by recurrent dynamics in prefrontal cortex," Nature, vol. 503, no. 7474, pp. 78-84, 2013.

[129] R. Cabeza, "Hemispheric asymmetry reduction in older adults: the HAROLD model," Psychology and Aging, vol. 17, no. 1, pp. 85-100, 2002.

[130] J. Domínguez-Borràs, J. L. Armony, A. Maravita, J. Driver, and P. Vuilleumier, "Partial recovery of visual extinction by pavlovian conditioning in a patient with hemispatial neglect," Cortex, vol. 49, no. 3, pp. 891-898, 2013.

[131] I. H. Robertson, "A noradrenergic theory of cognitive reserve: implications for Alzheimer's disease," Neurobiology of Aging, vol. 34, no. 1, pp. 298-308, 2013.

[132] R. C. Aster, B. Borchers, and C. H. Thurber, Parameter Estimation and Inverse Problems, Elsevier, 2nd edition, 2012.

[133] G. J. Goodhill and H. G. Barrow, "The role of weight normalization in competitive learning," Neural Computation, vol. 6, no. 2, pp. 255-269, 1994.

[134] D. Neumann, Connectivity of the brain from magnetic resonance imaging [Ph.D. dissertation], California Institute of Technology, 2010, http://resolver.caltech.edu/CaltechTHESIS:04282010153942989.

[135] G. Tononi and C. Cirelli, "Sleep function and synaptic homeostasis," Sleep Medicine Reviews, vol. 10, no. 1, pp. 49-62, 2006.

[136] D. Rudrauf, A. Lutz, D. Cosmelli, J.-P. Lachaux, and M. Le Van Quyen, "From autopoiesis to neurophenomenology: Francisco Varela's exploration of the biophysics of being," Biological Research, vol. 36, no. 1, pp. 27-65, 2003.

[137] S. Dehaene, M. Kerszberg, and J.-P. Changeux, "A neuronal model of a global workspace in effortful cognitive tasks," Proceedings of the National Academy of Sciences of the United States of America, vol. 95, no. 24, pp. 14529-14534, 1998.
[138] F. Varela, J.-P. Lachaux, E. Rodriguez, and J. Martinerie, “The brainweb: phase synchronization and large-scale integration," Nature Reviews Neuroscience, vol. 2, no. 4, pp. 229-239, 2001.

[139] K. Friston, "The free-energy principle: a rough guide to the brain?” Trends in Cognitive Sciences, vol. 13, no. 7, pp. 293-301, 2009.

[140] K. Friston, "The free-energy principle: a unified brain theory?" Nature Reviews Neuroscience, vol. 11, no. 2, pp. 127-138, 2010.

[141] M. V. J. Veenman, Intellectual Ability and Metacognitive Skill: Determinants of Discovery Learning in Computerized Learning Environments, University of Amsterdam, 1993.

[142] K. Williford, D. Rudrauf, and G. Landini, "The paradoxes of subjectivity and the projective structure of consciousness," in Consciousness and Subjectivity, S. Miguens and G. Preyer, Eds., Ontos, Frankfurt, Germany, 2012.

[143] D. A. Stanley and R. Adolphs, "Toward a neural basis for social behavior," Neuron, vol. 80, no. 3, pp. 816-826, 2013.

[144] J. K. O’Regan and A. Noë, “A sensorimotor account of vision and visual consciousness," Behavioral and Brain Sciences, vol. 24, no. 5, pp. 939-1031, 2001.

[145] D. Rudrauf, A. Lutz, D. Cosmelli, J.-P. Lachaux, and M. Le Van Quyen, "From autopoiesis to neurophenomenology: Francisco Varela's exploration of the biophysics of being," Biological Research, vol. 36, no. 1, pp. 27-65, 2003.

[146] R. Goldberg, "Survey of virtual machine research," IEEE Computer, vol. 7, no. 6, pp. 34-45, 1974.

[147] D. C. Dennett, Consciousness Explained, Little, Brown and Company, Boston, Mass, USA, 1991.

[148] T. J. Sejnowski, C. Koch, and P. S. Churchland, "Computational neuroscience," Science, vol. 241, no. 4871, pp. 1299-1306, 1988.

[149] P. S. Churchland and T. J. Sejnowski, The Computational Brain, MIT Press, Cambridge, Mass, USA, 1992.

[150] J. von Neumann, First Draft of a Report on the EDVAC, United States Army Ordnance Department, University of Pennsylvania, 1945.

[151] W. S. McCulloch and W. Pitts, "A logical calculus of the ideas immanent in nervous activity," The Bulletin of Mathematical Biophysics, vol. 5, no. 4, pp. 115-133, 1943.

[152] Y. He, L. Wang, Y. Zang et al., "Regional coherence changes in the early stages of Alzheimer's disease: a combined structural and resting-state functional MRI study," NeuroImage, vol. 35, no. 2, pp. 488-500, 2007.

[153] N. Villain, B. Desgranges, F. Viader et al., "Relationships between hippocampal atrophy, white matter disruption, and gray matter hypometabolism in Alzheimer's disease," Journal of Neuroscience, vol. 28, no. 24, pp. 6174-6181, 2008.

[154] L. A. Beckett, D. J. Harvey, A. Gamst et al., “The Alzheimer's Disease Neuroimaging Initiative: annual change in biomarkers and clinical outcomes," Alzheimer's and Dementia, vol. 6, no. 3, pp. 257-264, 2010.

[155] Y. Li, Y. Wang, G. Wu et al., "Discriminant analysis of longitudinal cortical thickness changes in Alzheimer's disease using dynamic and network features," Neurobiology of Aging, vol. 33, no. 2, pp. 427.e15-427.e30, 2012.

[156] D. T. Jones, M. M. MacHulda, P. Vemuri et al., "Age-related changes in the default mode network are more advanced in Alzheimer disease," Neurology, vol. 77, no. 16, pp. 1524-1531, 2011.

[157] O. Lazarov, J. Robinson, Y.-P. Tang et al., "Environmental enrichment reduces $\mathrm{A} \beta$ levels and amyloid deposition in transgenic mice," Cell, vol. 120, no. 5, pp. 701-713, 2005. 
[158] S. M. Smith, P. T. Fox, K. L. Miller et al., "Correspondence of the brain's functional architecture during activation and rest," Proceedings of the National Academy of Sciences of the United States of America, vol. 106, no. 31, pp. 13040-13045, 2009.

[159] P. Goel, A. Kuceyeski, E. Locastro, and A. Raj, "Spatial patterns of genome-wide expression profiles reflect anatomic and fiber connectivity architecture of healthy human brain," Human Brain Mapping, 2014. 

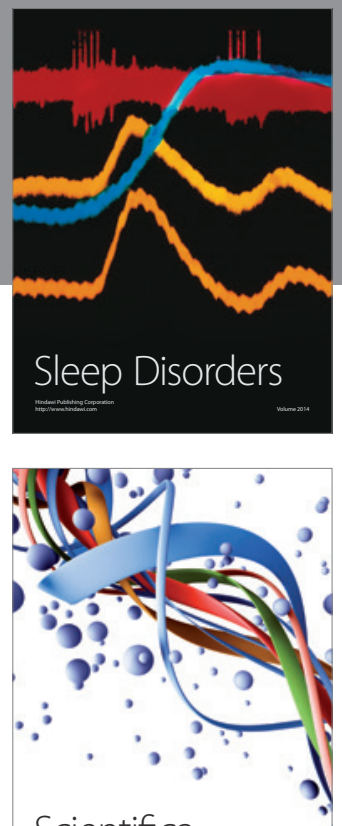

Scientifica
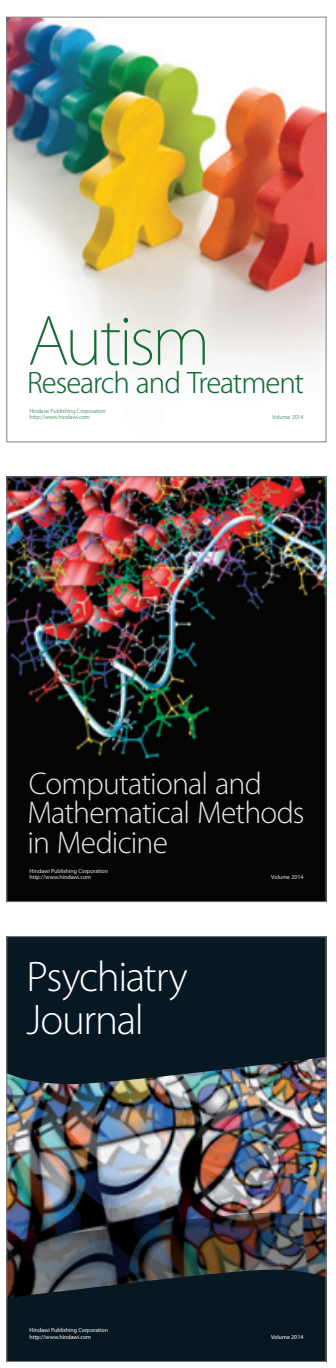
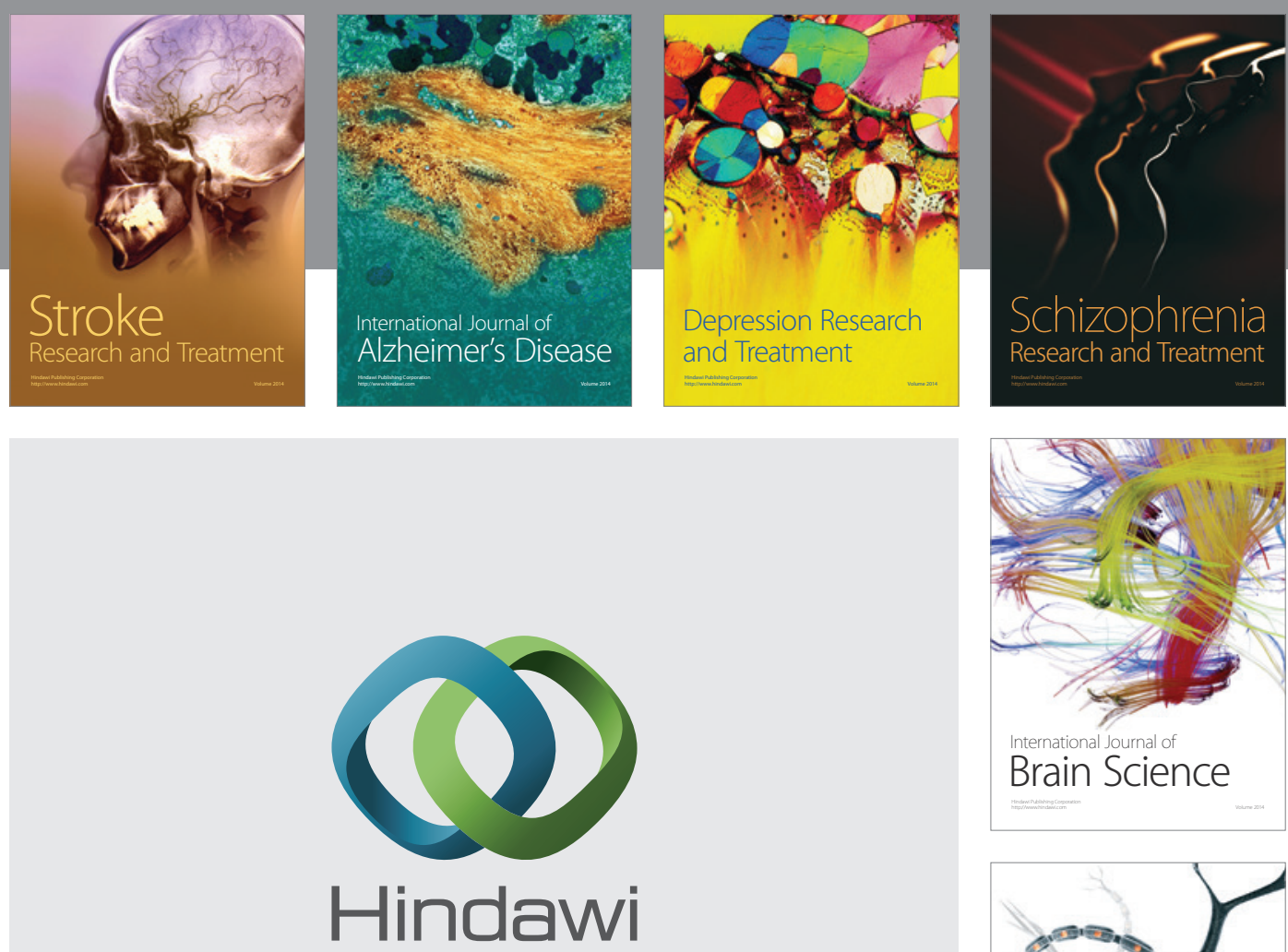

Submit your manuscripts at

http://www.hindawi.com
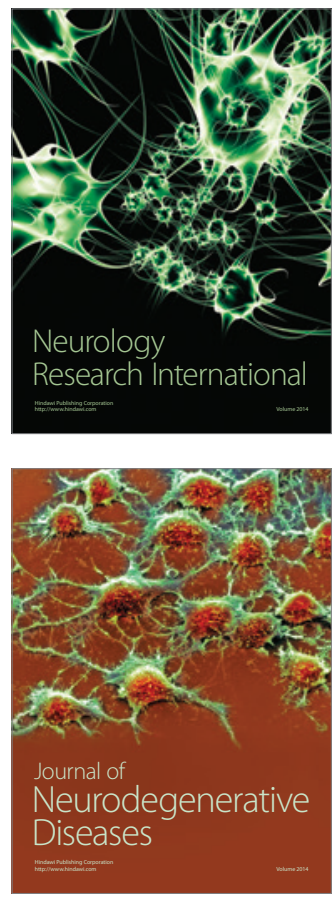

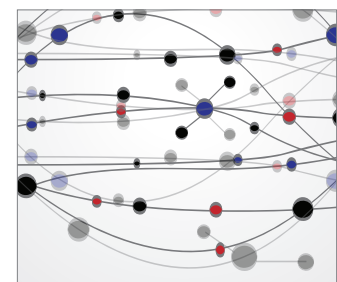

The Scientific World Journal
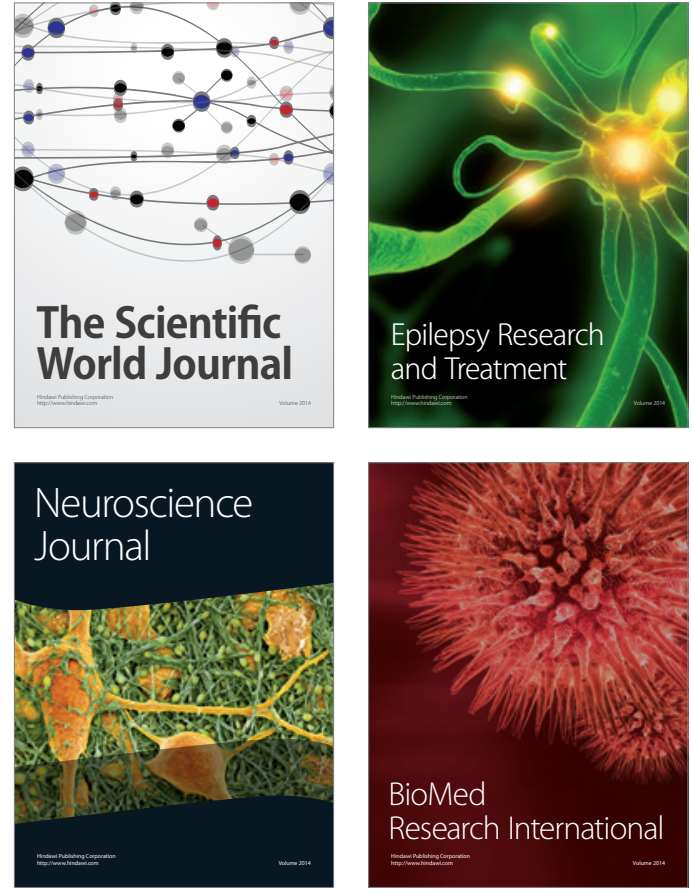

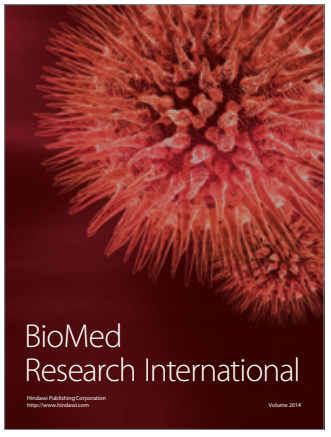

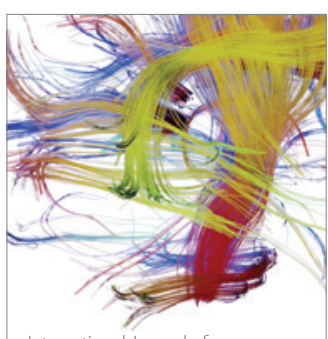

Brain Science

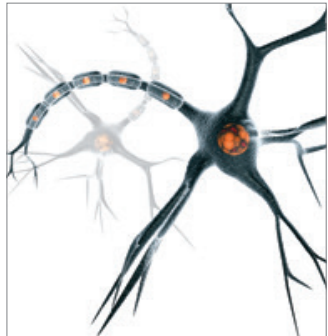

Neural Plasticity
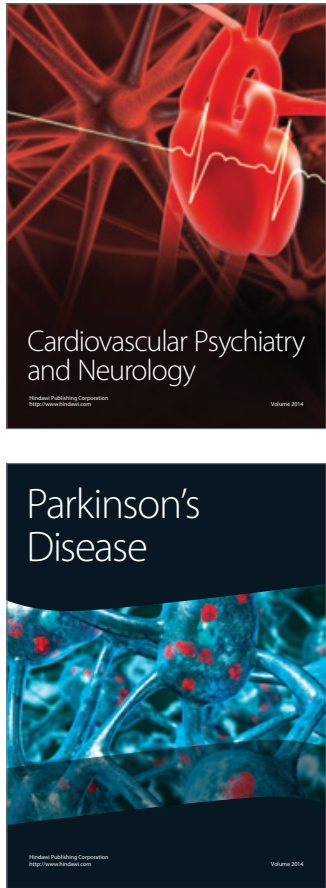